\title{
Advances in Tribology of Lubricin and Lubricin-Like Synthetic Polymer Nanostructures
}

\author{
Ilker S. Bayer ${ }^{(D)}$ \\ Smart Materials, Istituto Italiano di Tecnologia, Via Morego 30, 16163 Genova, Italy; ilker.bayer@iit.it; \\ Tel.: +39-380-387-6699
}

Received: 2 January 2018; Accepted: 15 March 2018; Published: 4 April 2018

\begin{abstract}
Articular cartilage surrounds the ends of diarthrodial joints (most common movable joints) and during motion, it experiences a wide range of loading conditions while remaining under exceedingly low-friction and wear-free conditions. This remarkable tribological performance stems from complex interactions between the synovial fluid and articular cartilage. In fact, lubricin and hyaluronic acid (HA) that are part of the synovial fluid are now known to be the key contributors to effective joint lubrication and wear protection. Studies involving animal models and artificial systems suggest that lubricin and HA molecules may work in tandem to produce a highly synergistic effect for lubrication. However, latest observations suggest that lubricin has significant potential for protecting the articular joints, probably more than HA. Recently, lurbicin-related friction regulation in soft eye tissues, where much lower forces are involved compared to knee joints for instance, has been shown to be related to dry eye disease and contact lens discomfort. As such, lubricin's role in natural friction regulation is very complex. Moreover, partially unresolved water-lubricin interactions are essential for lubrication and load carrying function in the joints. The chemical structure of lubricin has inspired several chemists to synthesize new copolymers and polymer brushes that function just like lubricin in order to design new synthetic or bio-based lubricants with ultra-low-friction coefficients. Hence, lubricin has emerged as a key natural molecule for bioinspired tribology. The aim of this review is to present the latest advances in understanding of lubricin's function in joint lubrication and in soft tissue friction (i.e., human eye) and document what has been achieved so far in transforming this biomedical knowledge into new polymer design for advanced engineering tribology. It is hoped that this review will catalyze research and development efforts in obtaining very stable and high load-bearing polymer-based ultra-low-friction surfaces via biomimicry.
\end{abstract}

Keywords: lubricin; biotribology; osteoarthritis; hyaluronic acid; polymer brush; dry eye

\section{Introduction}

Nature has been designing lubricants and adhesives for millions of years. Scientists are now capable of gathering information about biological surfaces in relative motion, their friction, adhesion, lubrication, and wear and have been able to relate this knowledge to technological applications and to the development of new-generation lubricants [1-7]. Miniaturization of many technological devices (i.e., data storage and management components) that we use today requires a fundamental understanding of tribological phenomena at the micro- and nano-metric scales. Biological systems also excel at these scales and might serve as templates for new-generation low-friction technologies. Examples of biosystems with unique tribological properties include articular cartilage, adaptive adhesion of white blood cells over blood vessel cell layers in response to inflammatory signals, and diatoms with rigid segments in relative motion, all of which have been engineered throughout evolution. In other words, evolution shows us that certain proteins such as collagen found in bones, skin, tendons, and the cornea can function in completely different modes (as an energy source or as 
a lubricant) due to its different organization and structuring optimized for different body locations and length scales [8]. In fact, the work by Gebeshuber et al. [8] does an excellent job in demonstrating these biological concepts pertaining to lubrication in living joints.

There are several materials that have been proposed as coatings or replacements for hips or joints etc. [9]. For instance, diamond-like carbon [10], hydrogels [11], and ultra-high molecular-weight polyethylene [12] are still being actively researched and improved for artificial joints. However, a synthetic biocompatible material mimicking cartilage dynamics can be beneficial toward repairing local cartilage defects less invasively compared to joint replacement to prolong life of an injured joint. As such, one needs to understand how the lubrication occurs and what natural macromolecules are involved in living joint tribology. The first and foremost lubricant of interest is the glycoprotein lubricin $[13,14]$. It is the primary boundary lubricant of articular cartilage. Its boundary lubricating abilities arise from two key structural features: (i) a dense mucin-like domain consisting of hydrophilic oligosaccharides and (ii) an end terminus that anchors the molecule to articulating surfaces. When bound, lubricin molecules attract and trap water near a surface, reducing friction and facilitating glide. It is postulated that bio-based polymers having structures similar to the block-co-polymer-like domains of lubricin would highly enhance lubrication and adhesion to articular cartilage. Indeed, synthetic analogues have been designed to mimic lubricin using, for instance, thiol-terminated polyacrylic acid-graft-polyethylene glycol (pAA-g-PEG) brush copolymers. The PEG moiety was designed to mimic the mucin-like domain of lubricin, and the thiol-terminus was designed to anchor the molecules to cartilage surfaces, mimicking the binding domain [15].

The aim of this review article is to present recent advances in tribological studies focusing on polymeric brushes, polymer nanoscale structures, and coatings that are designed to mimic lubricin as well as the friction dynamics in synovial fluid constrained between closely spaced surfaces. It is beyond doubt that many other exciting material systems, such as hydrogels, graphene and its composites, and titanium-based coatings, are being developed to address medical problems associated with osteoarthritis. Herein, the author will exclude such advances targeting biotribology applications due to lack of space and limit his attention to macromolecular assemblies originating from natural or synthetic lubricin-like polymeric constructs. For advances in aforementioned alternative materials, the readers can refer to a number of excellent articles [16-20]. In the following, the author will first define lubricin and briefly present its chemical form and demonstrate how biologists and medical doctors gauge its importance in bone and joint related diseases. Then, a review of recent works on the measurement and simulation of tribological behavior of lubricin in biomedical environments including soft tissues such as eyelids will be presented, and finally, lubricin-like co-polymer/protein constructs in the form of coatings that resemble synovial fluid lubrication will be discussed along with how these new materials help us improve tribology of both biologically and industrially relevant surfaces.

\section{Definition, Function, and Structure of Lubricin and Its Bioinspiration Potential}

One of the most widely used synthetic low-friction coefficient materials for bearings is polytetrafluoroethylene (PTFE or Teflon) with a friction coefficient of approximately 0.04 (sphere on glass; speed: $10^{-6} \mathrm{~m} / \mathrm{s}$; load: $10 \mathrm{~N}$, roughness $<0.01 \mu \mathrm{m}$ ) [21]. Nature and biology, however, demonstrate even better low-friction coefficient materials. Natural joint mechanical properties are remarkably robust and complicated. Cartilage is glazed with a special polymer-like layer enabling joints to move practically friction-free, even under high loads. Our joints are lubricated by synovial fluids with very low-frictional properties. Both tribologists and medical scientists are still trying to understand the mechanisms underlying how load-bearing articulating cartilage surfaces, in contact with synovial fluid, experience friction coefficients close to 0.001 or less [22]. In fact, articular joints are lubricated by synovial fluid; if this lubrication is disrupted, it can result in osteoarthritis, which affects millions of people [23]. Moreover, even fish suffer from the same problem as recently demonstrated by Askary et al. [23]. They demonstrated that taking out the lubricin gene from the zebrafish genome causes arthritis in their jaws and fins. They identified bone sections (see Figure 1) where lubricin is 
present in order to facilitate jaw motion under water. Their findings showed that zebrafish can be a relevant model to understand the development of synovial specializations, including the poorly understood process of cavitation.
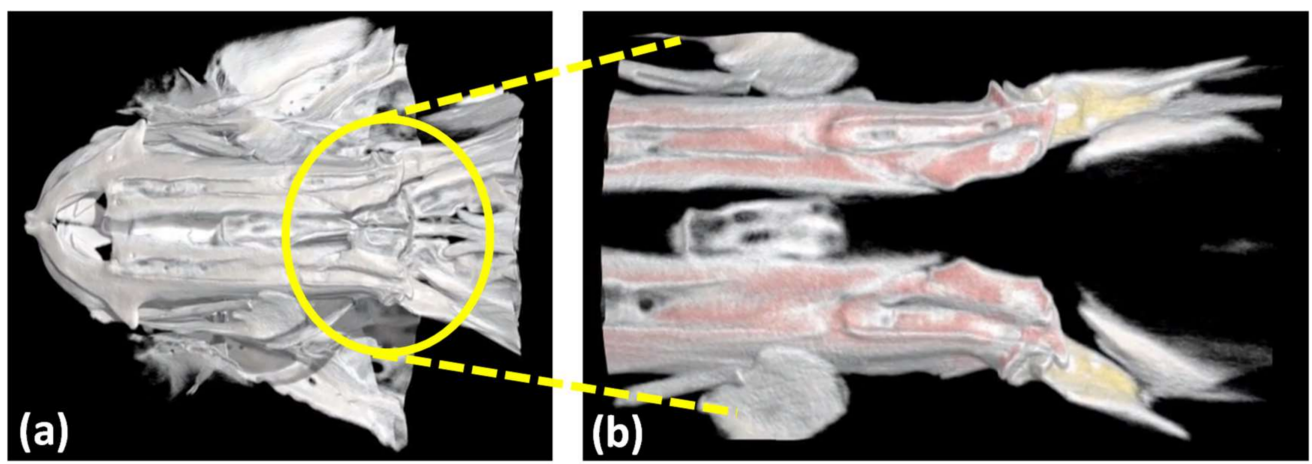

Figure 1. (a) Reconstructed adult zebrafish head in 3D. (b) Enlarged bone section shows the structure and relative position of the jaw joint. Colors in the enlarged reconstruct demonstrates the anguloarticular (pink) and quadrate (yellow) bones making up part of the jaw joint [23]. Copyright from eLife Sciences Publications, 2016.

Chemically, lubricin is a mucinous glycoprotein secreted in synovial joints [5]. In healthy joints, lubricin molecules form a coating over the cartilage surface, providing boundary lubrication and preventing cell and protein adhesion. Lubricin was initially identified in synovial fluid as a product of synovial cells and was labeled as superficial zone protein (SZP), which is a product of the superficial zone chondrocytes of articular cartilage, encoded by the PRG4 gene [13]. Later, lubricin was differentiated from SZP based on molecular weight differences, although some chemical differences were also noted $[14,24,25]$. It has been reported that observed differences between the molecular weight of lubricin $(230-280 \mathrm{kDa})$ and SZP $(345 \mathrm{kDa})$ can be attributed to the variance in the splicing of exons. Their similar molecular structure suggests that they both act as anti-adhesive lubricant proteins. The terms lubricin, SZP, and PRG4 are often used interchangeably to designate lubricin protein. Figure 2 demonstrates a schematic structure of lubricin with different molecular sections. Notice the block-copolymer-like structure.

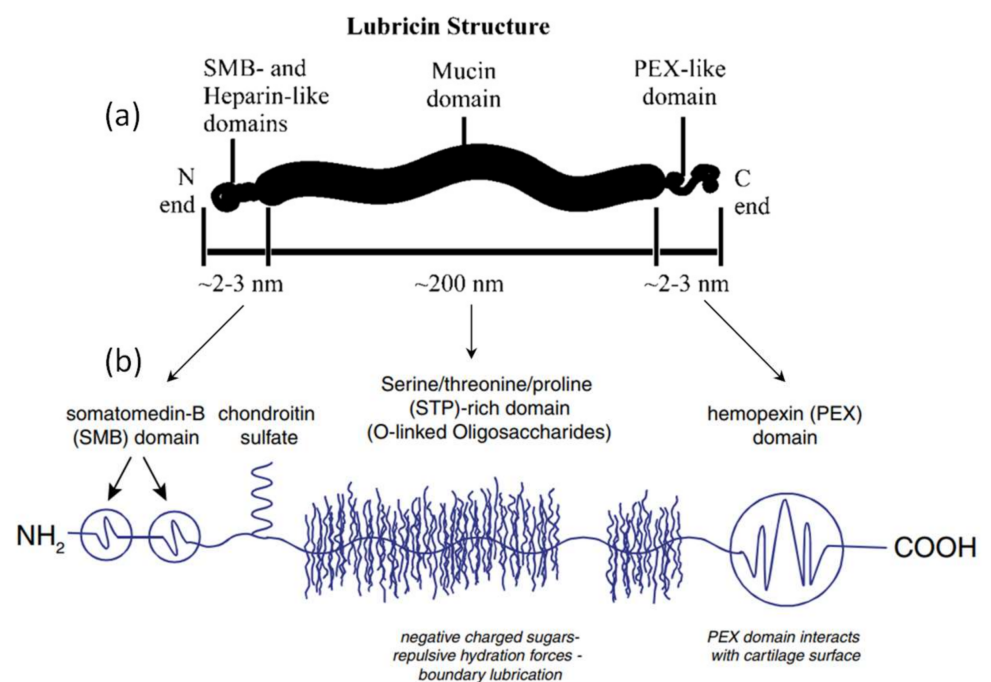

Figure 2. (a) Schematic structure of lubricin. Lubricin monomers have a 227-345 kDa molecular weight [24] Copyright from Elsevier, 2017. (b) Complex and detailed block-copolymer type structure of lubricin with functional end groups [25]. Copyright from Elsevier, 2007. 
Lubricin-assisted boundary lubrication takes place at very low sliding speeds when the lubricating film thickness is close to the surface roughness or asperities on the opposing cartilage surfaces. In the absence of lubricin, adhesion occurs increasing friction and producing a stick-slip phenomenon at the joint. At higher joint motions, such as running, the hydrodynamic regime takes control. The fluid filling the gap between surfaces moves at the same speed as the joint surface during movement. For instance, synovial fluid (SF) that enters a converging gap during motion will experience pressure increase as the gap converges; this in turns creates a hydrodynamic lift and forces the surfaces apart like a wedge. Preferably, the thickness of the film formed by the fluid should be slightly greater than the surface roughness in order to minimize asperity contact. It is now well understood that key bio-lubricants that work in synergy with lubricin include phospholipids and hyaluronan with an overall bottlebrush structure. In fact, this smooth motion of synovial joints due to lubricin and other biomaterials have intrigued scientists for years, inspiring work to understand the nano-mechanical characteristics of nano-textured cartilage surface, identify the mechanisms of lubrication important for the development of biomimetic lubricants. This can address the problem of finding the right materials for low friction and high load-bearing capacity between sliding surfaces in aqueous solutions. As an example, Banquy et al. [26] showed that molecular brushes with triblock polymer structures can form entangled loops when strongly adsorbed on a surface. Tribological properties of these polymers mimic the lubricating protein lubricin (see Figure 3), even when confined to a monolayer. The lubrication is attributed to strong osmotic repulsion between the side chains of the molecular brushes and the surfaces. This ensures the flow of water molecules during shear improving lubrication. They further showed that lubrication could be sustained independent from shearing speed and applied loads under molecular confinement.

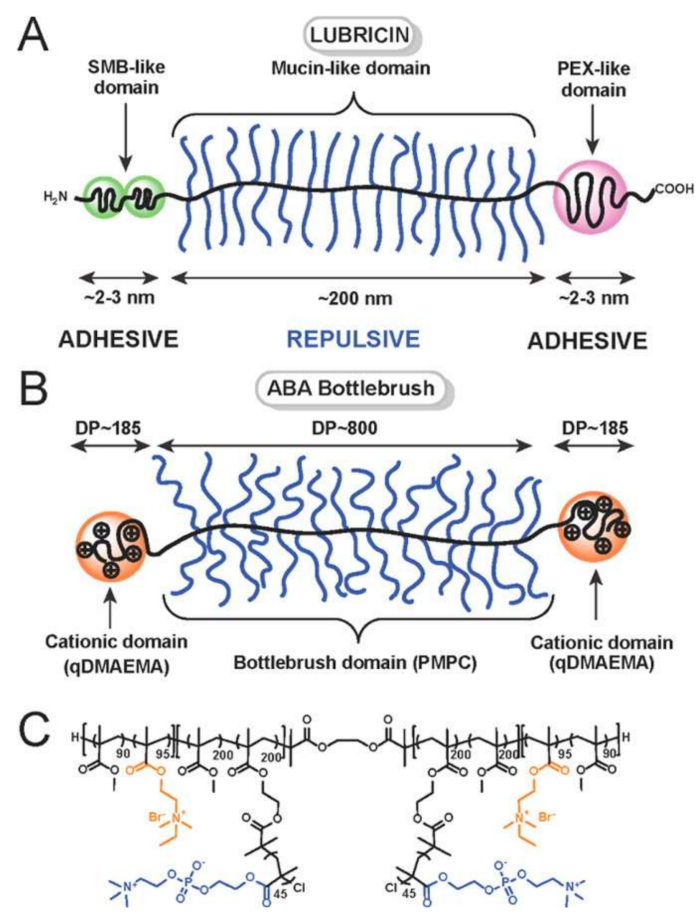

Figure 3. Schematic representations of the protein lubricin commonly encountered in mammalian synovial fluids (A). Structure of the bottlebrush polymer resembling lubricin (B,C) and polymeric morphology [26]. Copyright from ACS Publications, 2014.

It must be noted that, although lubricin is at the forefront of biotribology, the real molecular interactions between several constituents of the synovial fluid such as lubricin, hyaluronan (HA), serum albumin (SA), fibronectin, and the glycoprotein chitinase 3-like-1 (CH3L1) are all believed to contribute to joint lubrication [27]. As an example, important interactions and co-expression and 
co-localization of CH3L1 and lubricin in normal and osteoarthritic animal cartilage models have been unraveled recently [28], as shown in Figure 4. The figure shows that, on the right section of the schematic joint, the downregulation of lubricin results in articular cartilage that is rich in CH3L1 causing osteoarthritis. However, the downregulation of lubricin, regardless of the type of interactions with other synovial fluid components, seems to be the major source of deterioration of frictional properties of articular joints.

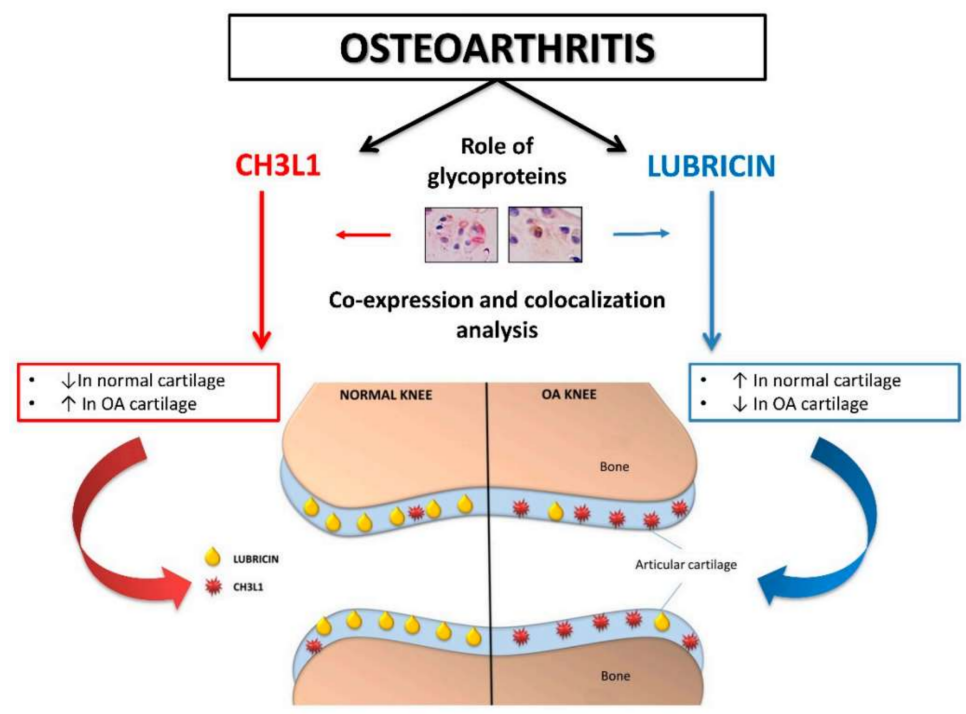

Figure 4. Schematic depicting the role of two glycoproteins (CHI3L1 and lubricin) in normal and osteoarthritic articular cartilage. Red arrows indicate CHI3L1 pathways and blue arrows show lubricin pathway [28]. OA: osteoarthritis, Copyright from authors, 2016.

In the following section, the author will review both experimental and theoretical understanding of biomedical lubrication taking place in moveable joints but also in soft tissues such as eyelids.

\section{Biomedical Aspects of Lubricin Mechanics and Tribology in Joints}

Lubricin is at the crossroads of biomechanics and biotribology [29]. Therefore, many medical conditions related to bone diseases or aging need to be correlated with the fluid mechanics and tribology of lubricin [30]. For instance, experiments on mice by Rhee et al. [31] indicated that, as the mice aged due to decreasing lubricin levels, some abnormal protein as well as cell deposits occurred on cartilage surfaces, causing the disappearance of underlying superficial zone chondrocytes (see Figure 5). In addition to cartilage surface changes and subsequent cartilage deterioration, intimal cells in the synovium surrounding the joint space became hyperplastic, which further contributed to joint failure. Purified or recombinant lubricin inhibited the growth of synovial fluid cells in vitro. They concluded that lubricin had multiple functions in articulating joints and tendons such as protection of surfaces and the control of synovial cell growth.

Jones et al. [32] studied molecular determinants facilitating lubricin to effectively bind to articular cartilage surfaces (Figure 6). Specific, localized, and efficient binding/adhesion to the surfaces was observed for synovial lubricin. Similar observations were also reported for recombinant full-length lubricin and a protein construct resembling lubricin C-terminal (hemopexin-like) domain (LUB-C, encoded by exons 7-12). Another construct resembling the N-terminal region of lubricin (LUB-N, encoded by exons 2-5) showed no significant binding/adhesion to cartilage, but instead started to dimerize, causing lubricin aggregation. They further showed that disulfide bond disruption strongly increased recombinant lubricin and LUB-C cartilage surface binding or adhesion. This indicates that protein secondary structure needs appropriate lubricin localization at pertinent tissue interfaces that is crucial for maintaining joint homeostasis (Figure 6). 

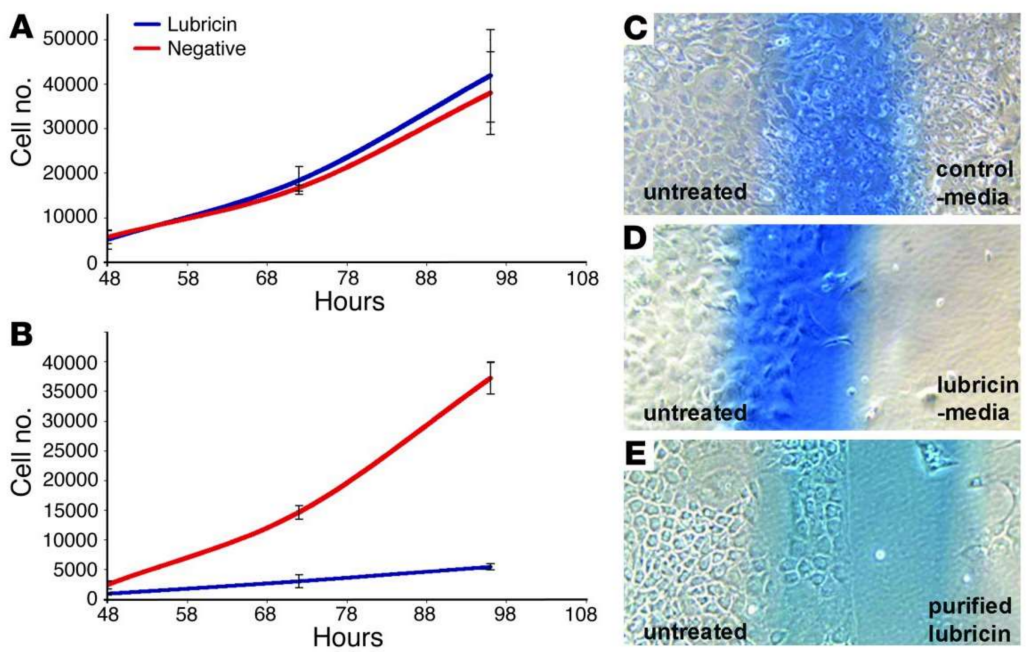

Figure 5. The effect of purified human lubricin on the in vitro growth of fibroblast-like synovial fluid cells. (A) Growth curves of adherent cells that were plated overnight in serum-containing media and then switched to growth media (red) or growth media with $60 \mu \mathrm{g} / \mathrm{mL}$ of purified lubricin (blue). (B) Growth curves of adherent cells that were plated overnight in serum-free media and then switched to growth media (red) or growth media with $60 \mu \mathrm{g} / \mathrm{mL}$ of purified lubricin (blue). When plates were not pre-exposed to serum-containing media, cell growth was significantly inhibited by purified lubricin. (C-E) Portions of tissue culture plates (to the right of the blue line) were incubated with control media, lubricin media, or $60 \mu \mathrm{g} / \mathrm{mL}$ of purified lubricin overnight. After washing off the media with several changes of PBS, cells were added to proliferate for 7 days. Few cells adhered to the lubricin-media or purified lubricin treated plates. Cells on the untreated portion of the plate did not proliferate onto the lubricin-treated portion [31]. Copyright from American Society for Clinical Investigation (ASCI), 2005.
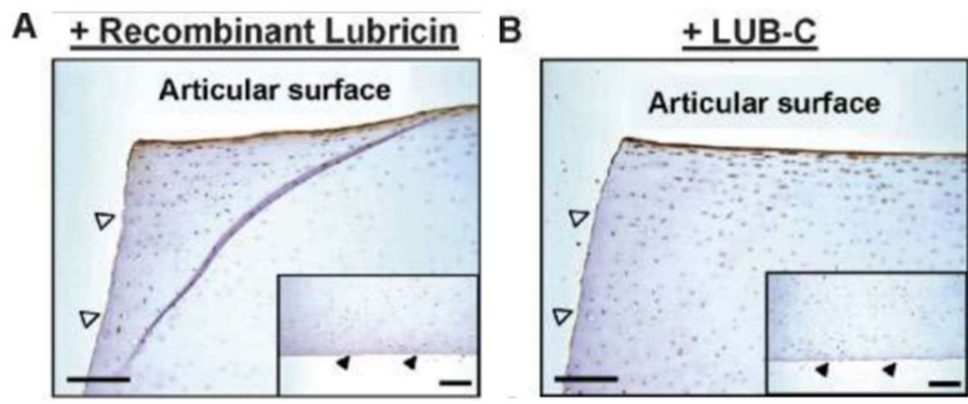

Figure 6. Images showing lubricin binding and localization. In particular, sagittal sections of extracted cartilage discs were incubated in the presence of (A) recombinant full-length lubricin and (B) LUB-C prior to immune-histochemical analysis with anti-lubricin anti-body 06A10 or anti-FLAG M2 antibody, respectively. Lubricin binding could be detected at articular surfaces, but not at cut sections or deeper zone surfaces (insets show open and closed arrowheads, respectively). Scale bar is $100 \mu \mathrm{m}$ [32]. Copyright from Wiley Online Library, 2007.

Increasing pressures within the interstitial fluid appears to be a primary frictional regulation mechanism response of articular cartilage [33]. However, synovial joints are not constantly filled with an ideal thickness fluid layer. In other words, boundary lubrication is a key process in the friction of synovial joints. As such, boundary lubricants can complement this important role of interstitial fluid pressurization to help further reduce the coefficient of friction of cartilage. In order to demonstrate the fact that not only is the presence of lubricin and HA in synovial fluids required for proper joint function, but regulations in the fluid dynamics of synovial fluid also affect joints in tandem. For instance, $\mathrm{Xu}$ et al. [34] for instance, conducted a computational synovial fluid dynamics 
study of a normal temporomandibular joint (TMJ) during jaw opening. The numerical results are summarized in Figure 7.

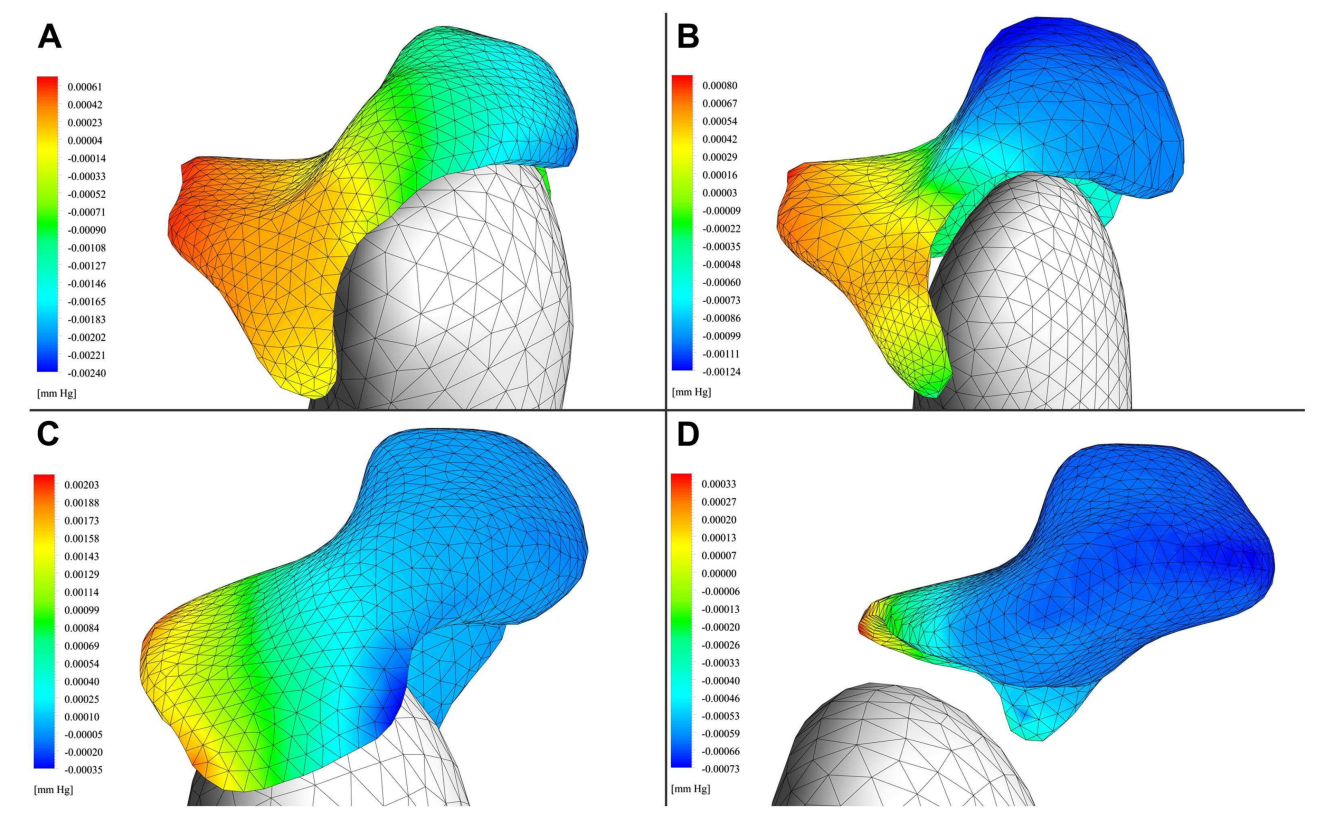

Figure 7. Simulation results of pressure distribution in the upper compartment of a normal TMJ. (A) A closed-jaw position. Jaw openings of (B) $1 \mathrm{~cm},(\mathbf{C}) 2 \mathrm{~cm}$, and (D) $3 \mathrm{~cm}$. TMJ = temporomandibular joint [34]. Copyright from Elsevier, 2013.

For a closed-jaw position, the authors demonstrated that the upper joint compartment conforms to a dumbbell shape. As the jaw opens, the frontal part of the upper compartment diminished gradually and disappeared completely when the jaw opened. During jaw opening, the pressure in the anterior space of the upper compartment was higher than that in the posterior space (see Figure 7). Their model indicated that synovial fluid circulates in an anticlockwise direction forming local vortices in both frontal and rear sections. As the joint disc moved, the synovial fluid circulates in an anticlockwise direction with the formation of local vortices.

Waller et al. [35] demonstrated a direct link between lubricin, boundary lubrication, and cell survival. They found that supplementation of synovial fluid with lubricin was an effective treatment to prevent cartilage deterioration in osteoarthritis. They studied the relationship between friction and chondrocyte death via ex vivo and in vitro measurements of friction and apoptosis and reported increases in joint friction and cellular apoptosis in lubricin knockout mice compared to wild-type mice. Upon utilizing an in vitro bovine explant cartilage-on-cartilage bearing system, a direct correlation between friction coefficient and chondrocyte apoptosis was demonstrated in cartilage superficial layers. Addition of lubricin as a test lubricant significantly lowered the static coefficient of friction and the number of apoptotic chondrocytes in the bovine explant system, as shown in Figure 8.

Wong et al. [36] tested the hypothesis that, during tibio-femoral articulation (Figure 9), cartilage lubrication by synovial fluid (including lubricin) extracted from acutely injured joints elevates tissue shear deformation, while lubrication by synovial fluid extracted from acutely injured joints with augmented lubricin-like hyaluronan reduces shear deformation toward normal magnitudes. They presented a detailed study in which, during tibio-femoral cartilage articulation, (1) the effects of acute injury on synovial fluid lubricant function and (2) the ability of lubricin-like hyaluronan addition to the synovial fluid of acutely injured joints to enhance lubricant function, with lubricant function, were assessed in terms of peak cartilage shear deformation during sliding motions. Acute injury impairs lubricin function, elevating cartilage Exz (shear stress on the XZ plane; see Figure 9) markedly 
during tibio-femoral articulation; such elevated Exz may contribute to post-injury associated cartilage degeneration. Since lubricin-like hyaluronan partially restores the function of the synovial fluid of acutely injured joints, as indicated by Exz, tribo-supplements may be beneficial in modulating normal cartilage homeostasis.

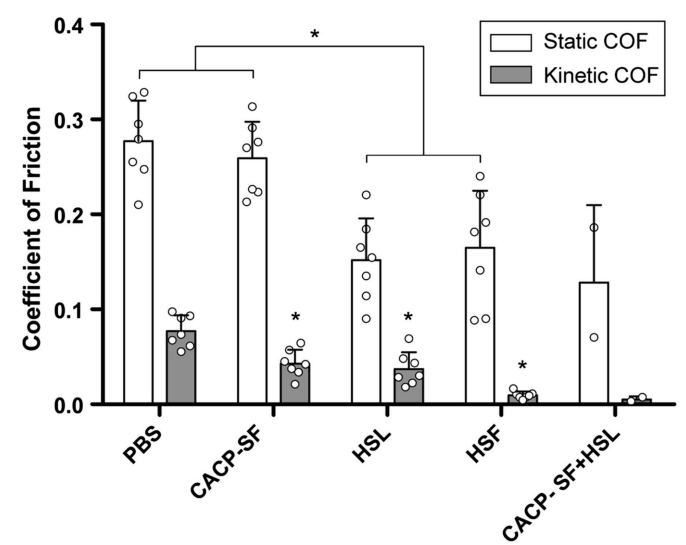

Figure 8. Friction coefficient (COF) results pertaining to bovine cartilage bearings lubricated with different test solutions. COF measurements are depicted as bar graphs with mean +1 SD static (unshaded) and kinetic (shaded) lubricated with either PBS, synovial fluid (SF) from patients with CACP (CACP-SF), purified human synoviocyte lubricin (HSL), human synovial fluid from normal joints (HSF), and CACP-SF supplemented with HSL (CACP-SF + HSL). Static values of COF were measured higher in PBS and CACP-SF in bearings compared to HSL- and HSF-lubricated bearings. Kinetic COF was also higher in bearings lubricated with PBS compared with all other groups. The asterisks $\left(^{*}\right)$ above the bars or group of bars denote significance level between two groups of samples. The general concept is that if $p$-value (probability) between two samples is $<0.05$ one asterisk $\left(^{*}\right)$ is used [35]. Copyright from National Academy Sciences, 2013.
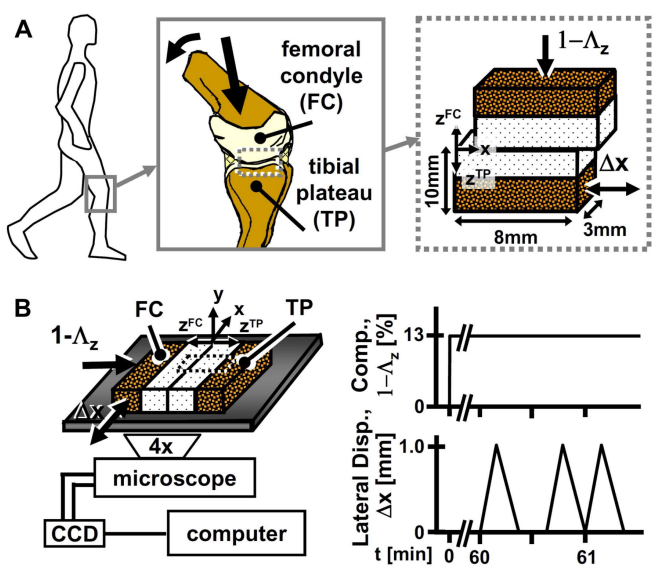

Figure 9. Schematic representation of (A) knee joint movement in different scales and (B) the microshear testing experimental setup and loading protocol. Loading variables are defined as compressive axial strain $(1-\Lambda z)$ and lateral displacement $(\Delta \mathrm{x})$ [36]. Copyright from Elsevier, 2010.

The experimental approach illustrated in Figure 9 can be explained as follows: Each lateral femoral condyle (FC) and tibial plateau (TP) pair was opposed in a custom bi-axial loading chamber mounted onto an epi-fluorescence microscope for digital video imaging (Figure 9B). The chamber secured the FC block at the bone and allowed in-plane movement of the opposing mobile TP block with orthogonally positioned plungers interfaced with either a micrometer (for axial displacement) or motion-controller (for lateral displacement). Subsequently, an axial displacement was applied 
$(\sim 40 \mu \mathrm{m} / \mathrm{s})$ to the bone portion of the FC sample to induce $13 \%$ compression $(1-\Lambda \mathrm{z}$, where $\Lambda \mathrm{z}$ is the stretch ratio) of the overall (i.e., FC and TP) cartilage thickness determined from gross images. Samples were then allowed to stress relax for $1 \mathrm{~h}$, which was confirmed to be sufficient to reach an approximate equilibrium stress for the sample geometries studied by Wong et al. [36]. Following axial compression, cartilage deformation was assessed separately in the FC and TP tissue during shear loading. Three sets of applied lateral displacements $(\Delta \mathrm{x})$, each consisting of $+1 \mathrm{~mm}$ and then $-1 \mathrm{~mm}$ (returning to initial position), were applied to the bone portion of the FC block (Figure 9B) at a rate of $0.1 \mathrm{~mm} / \mathrm{s}$ to induce relative lateral motion. The first set of sliding motion was for preconditioning, while tissue motions during the second and third set were recorded for analysis of TP and FC cartilage, respectively. Before and during the application of lateral displacements, sequential fluorescence images were taken separately for cartilage sections at increments of $33 \mu \mathrm{m}$ to assess shear deformation and sliding during tibio-femoral cartilage articulation.

Reesink et al. [37] studied reinforcement of lubricin boundary layer with galectin-3. This caused improvements in cartilage lubrication, and they argued that it could further delay the onset and advancement of arthritis. They reported that the lubricin glycome is enriched with terminal $\beta$-galactosides known as binding partners for some multivalent lectins called galectins. They examined the lubricin glycome to detect potential synovial fluid binding components for lubricin. Due to enrichment of terminal $\beta$-galactoside residues and the density of $\beta(1-3)$ Gal-GalNAc T-antigens in normal equine synovial fluid lubricin, along with the copious existence of galectins- 1 and -3 in cartilage and synovial fluid, they hypothesized that galectins can take part as binding sites for lubricin (Figure 10); as such, galectin-3 could be capable of enhancing boundary lubrication by cross-linking oligosaccharide structures on lubricin causing stabilization of the lubricin lattice.

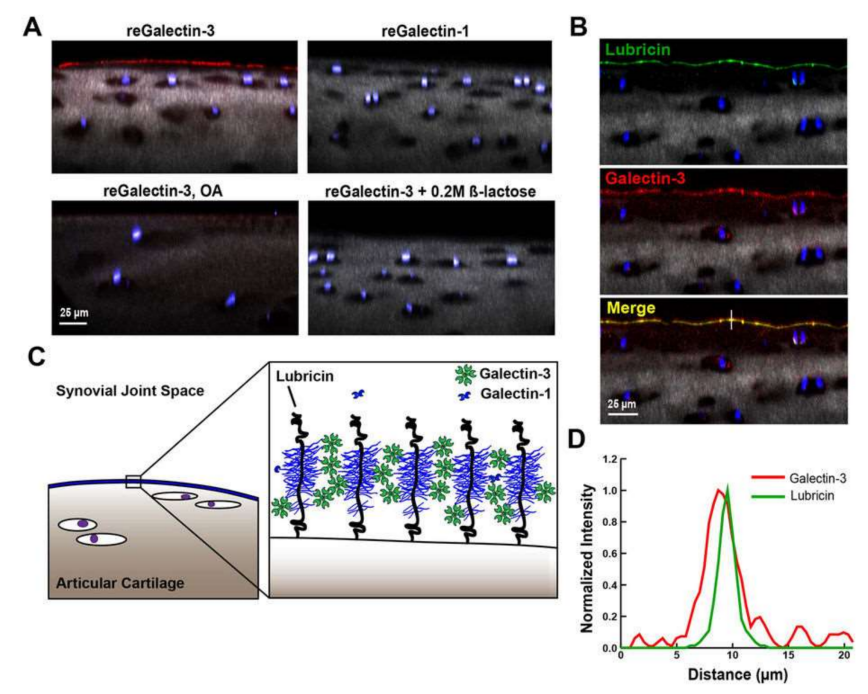

Figure 10. (A) Confocal and multiphoton microscopy $(40 \times)$ images of equine articular cartilage explants after incubation with Alexa647-conjugated galectins. Chondrocyte nuclei are stained with DAPI (4',6-diamidino-2-phenylindole; a fluorescent stain), and collagen is imaged with second harmonic generation microscopy (in gray). Galectin-3 prominently localizes at the boundary layer of healthy cartilage, whereas galectin-1 does not. Galectin-3 staining is significantly decreased in joints with severe osteoarthritis (OA) and in the presence of $0.2 \mathrm{M} \beta$-lactose, suggesting carbohydrate-specific binding. (B) Lubricin stained with anti-lubricin mAb MABT401 and A647-conjugated galectin-3 attached to the articular cartilage surface. Collective localization of lubricin and galectin-3 can be noticed. (C) Schematic mechanism depicting galectin-3 stabilized lubricin boundary layer. Pentavalent galectin-3 can bind to glycans on neighboring lubricin polymer brushes. This stabilizes the boundary layer mechanically via lubricin crosslinking. (D) Line scan from the vertical white line in B demonstrating lubricin and galectin-3 collective localization within the articular cartilage boundary layer [37]. Copyright from Springer-Nature, 2016. 


\section{Recent Advances in the Role of Lubricin in Dry-Eye-Related Treatments}

Lubricin has also attracted a significant amount of research related to dry-eye-related issues and the development of new-generation contact lenses [38] PRG4 glycoprotein (lubricin) is also produced by tear-secreting tissues found in the eye (work still continues on the relevant concentrations). Recently, micro-tribometers have been used in several studies in determining the friction coefficient of such tissues relevant to ophthalmology and contact lenses. Indeed, these setups can measure the right kind of frictional force resolutions by analyzing microscopic samples and applying biologically relevant normal force ranges. Lubricin may function by adsorbing to biological surfaces from tear fluid and form a hydrated, low-friction boundary layer. In other words, the N- or C-terminals of the protein domains of lubricin can anchor the molecule to a surface, leaving a negatively charged, hydrophilic mucin layer that creates electrostatic and steric repulsion. On the other hand, HA is already used in commercial eye drops and contact lens solutions [39]. A human recombinant PRG4 is also now available (Lubris BioPharma LLC, Framingham, MA, USA) that shows promise for clinical application [39].

Samsom et al. [40] used in vitro friction testing with human cadaver tissues (human corneas; age: 54-81). Their purpose was to (i) determine if lubricin can sorb to and lubricate model contact lens materials and (ii) evaluate the boundary lubricating ability of lubricin and HA compared to saline on model contact lens materials. The materials used in their study were restricted to common contact lens hydrogels polyhydroxyethylmethacrylate (pHEMA), N,N-dimethylacrylamide (DMAA), and Tris-(trimethyl-silyl-propyl-methacrylate) (TRIS).

Their results indicated that there is a synergy between HA and lubricin lubrication particularly for acrylic contact lenses and cornea that enhances comfort levels of contact lens usage for very dry eyes. Their measurements are given in Figure 11. In another study [39], following a two-week clinical trial in which the effects of lubricin and sodium hyaluronate (HA) were compared, patients suffering from moderate dry eye disease reported significant improvements and diminishing related disease signs and symptoms [39]. Refer to Figure 12 for detailed clinical results. Recombinant human lubricin $150 \mu \mathrm{g} / \mathrm{mL}$ was found to be safe, well tolerated, and generally superior to $0.18 \%$ sodium hyaluronate, improving both signs and symptoms in subjects with moderate dry eye disease.
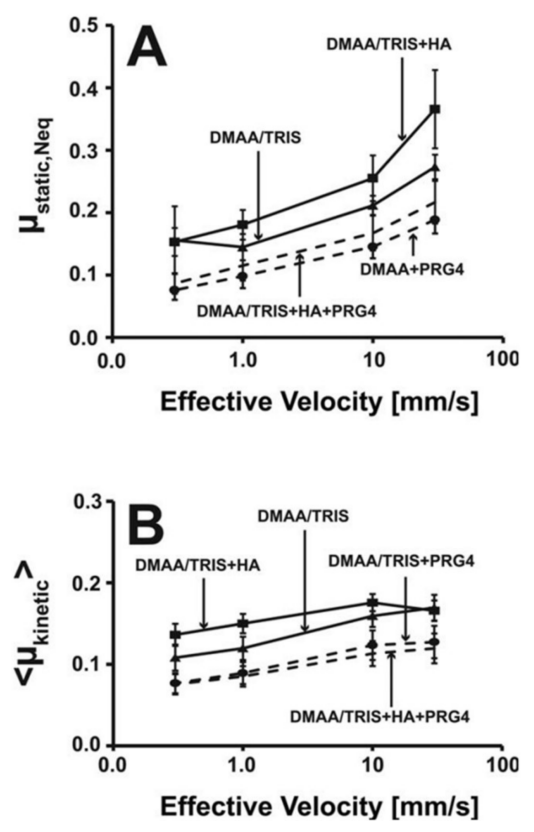

Figure 11. Effects of lubricin and HA on boundary lubrication at a human cornea-DMAA/TRIS biointerface. Static, $\mu_{\text {static }}(\mathbf{A})$, and kinetic, $\mu_{\text {kinetic }}($ B) friction coefficients $(\mu)$ measured in baths of saline, lubricin (PRG4) at $300 \mu \mathrm{g} / \mathrm{mL}$ in saline, $\mathrm{HA}$ at $1.2 \mathrm{mg} / \mathrm{mL}$ in saline, or HA and lubricin in saline at these concentrations [40]. Copyright from Wiley Online Library, 2017. 


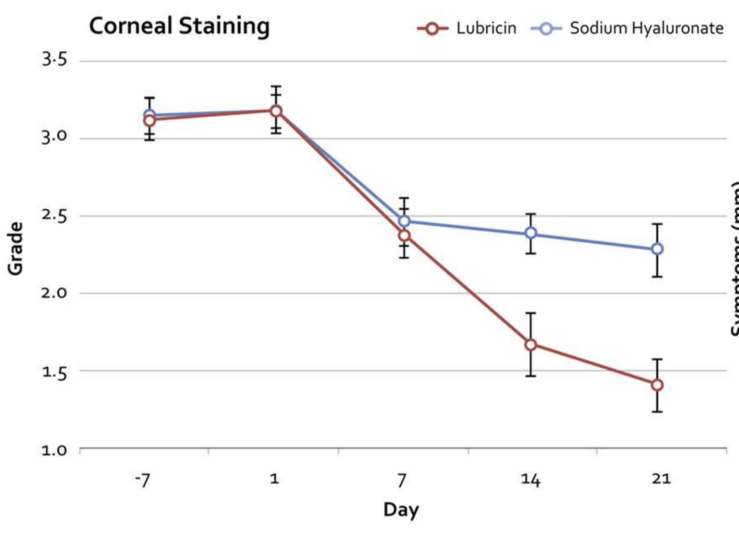

(a)

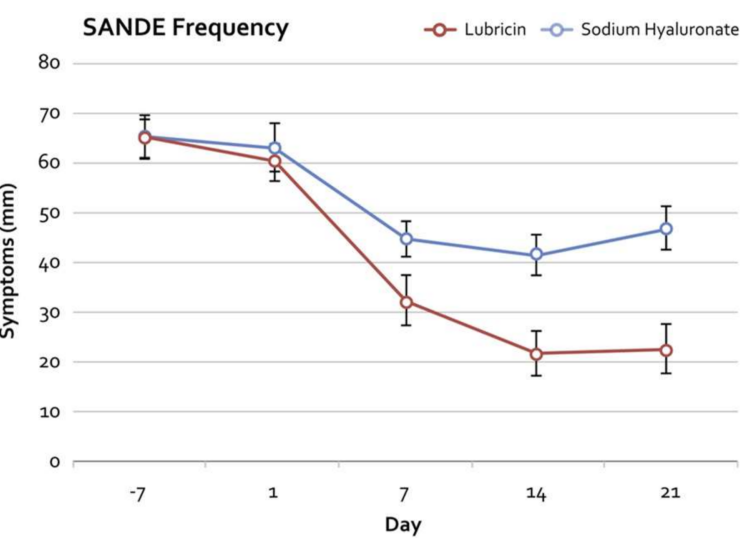

(b)

Figure 12. (a) Treatment effects of lubricin and sodium hyaluronate on corneal fluorescein staining. Results reported as the average of the two eyes of each patient \pm SEM. After the as-needed (PRN) phase of the study on Day 14, the 1.5 grade, the $46.9 \%$ reduction in staining in the lubricin group almost doubled the 0.8 grade, and a $24.9 \%$ reduction from baseline observed with HA therapy $(p<0.04)$. (b) Symptom assessment in dry eye (SANDE) frequency and lubricin. After the PRN phase of the study on Day 14 , the $61.3 \%$ reduction in symptoms in the lubricin group almost doubled [39]. Copyright from Elsevier, 2017.

Morrison et al. [41] designed a study to implement and characterize an in vitro boundary lubrication test at a human cornea-polydimethylsiloxane (PDMS) biointerface and to determine the dose-dependent and synergistic effects of lubricin, with HA, on ocular surface boundary lubrication using this test. They used human corneas and model PDMS material that were articulated against each other, at effective sliding velocities between 0.3 and $30 \mathrm{~mm} / \mathrm{s}$ under physiologic loads of approximately 8-25 kPa. Samples were tested serially either (1) in saline, in lubricin at 30, 100, and $300 \mu \mathrm{g} / \mathrm{mL}$ re-suspended in saline, and then in saline again or (2) in saline, in a commercial eye drop solution (containing 0.1\% HA), in $300 \mu \mathrm{g} / \mathrm{mL}$ PRG4 in saline, in $300 \mu \mathrm{g} / \mathrm{mL}$ PRG4 in the eye drop solution, and then saline again. They calculated both static and kinetic friction coefficients. They found that lubricin effectively lowered friction at the cornea-PDMS biointerface, both alone in a dose-dependent manner and in combination with HA. Lubricin also reduced kinetic friction coefficients and it functioned as an effective ocular surface boundary lubricant, both alone in a dose-dependent manner and in combination with HA.

Again, Samsom et al. [42] performed in vitro friction tests (see Figure 13) to measure and study the friction coefficients of silicone hydrogel ( $\mathrm{SiHy}$ ) contact lenses against both human eyelid and cornea tissues. They reported that lubricin was an effective boundary lubricant for $\mathrm{SiHy}$ lenses at both cornea and eyelid biointerfaces. Lubricin significantly reduced friction during two-week usage of SiHy lenses made from senofilcon $A$ against both eyelids and the cornea. Moreover, lubricin significantly reduced friction for daily disposable narafilcon $A$ and delefilcon $A$ daily lenses in saline alone. They confirmed that recently available recombinant human lubricin can have clinical utility in reducing friction of contact lenses, either as a topically applied lubricant or as a covalent link to the lens surface, therefore improving in vivo comfort.

Schmidt et al. [43] tested the hypotheses that lubricin is produced by ocular surface epithelia and protects the cornea and conjunctiva against significant shear forces generated during an eyelid blink and that lubricin deficiency increases shear stress on the ocular surface and promotes corneal damage. Their results indicated that lubricin is transcribed, translated, and expressed by ocular surface epithelia and demonstrated that lubricin presence significantly reduced friction between the cornea and conjunctiva and that lubricin deficiency may play a role in promoting corneal damage. They found that the measured friction coefficient $(\mu)$ is always lower when lubricin is present in the tissue. 


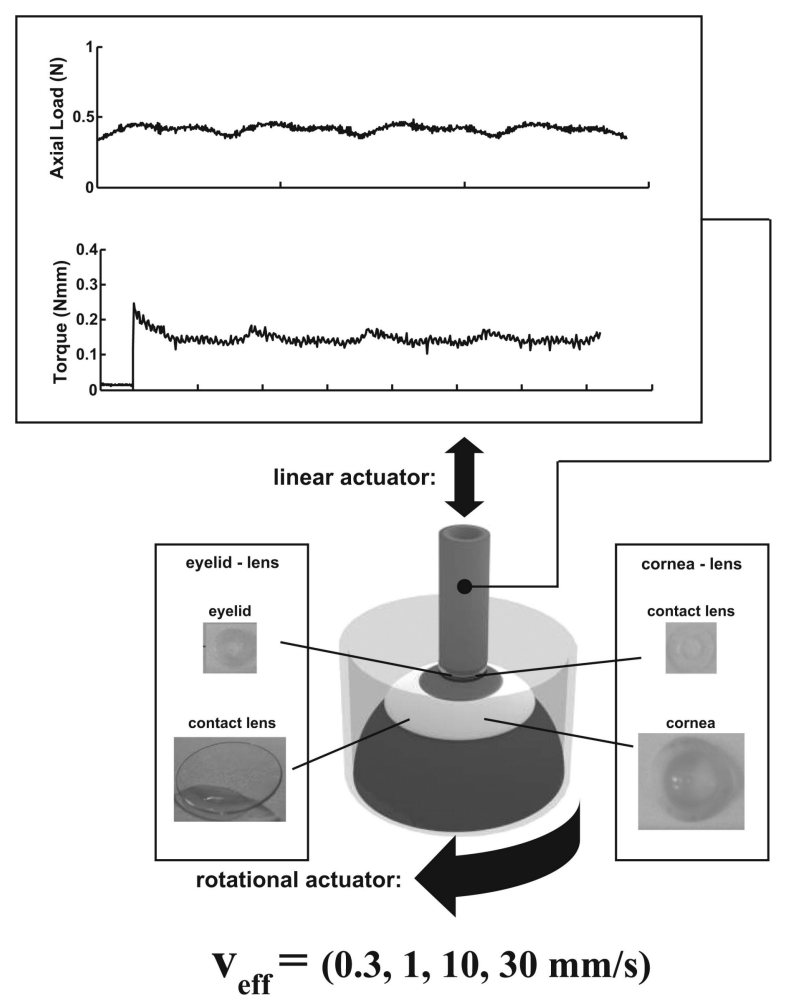

Figure 13. Schematic of in vitro friction test setup used by Samsom et al. [42]. Copyright from Elsevier, 2015. Example tissue samples, contact lens samples are tested and raw axial load and torque data recorded (top panels).

Similarly, a very recent study Regmi et al. [44] investigated enzymatic interactions of lubricin in the eye and showed that dry-eye-disease-related enzymatic breakdown of lubricin causes significant discomfort in patients due to increased friction coefficients in human eyes (see Figure 14). The objective of their study was to assess the ability of cathepsin S (CTSS, a class of cysteine proteinases that can be secreted into the extra-cellular space and can further activate MMP proenzymes) to enzymatically degrade purified lubricin, as well as lubricin naturally present in human tears, and to alter the ocular surface boundary lubricating properties. Note that MMP proenzymes are matrix metalloproteinases, also known as matrixins. They are mainly calcium-dependent zinc-containing endopeptidases. Figure 14a shows that the kinetic friction coefficient of human eyelid is minimum when lubricin is enzyme-free (rhPRG4). In Figure 14b, both polyacrylamide gel electrophoresis (PAGE-separation of biological macromolecules, usually proteins or nucleic acids, according to their electrophoretic mobility) and the western blotting (sometimes called the protein immunoblot; an antibody staining process) techniques demonstrate that CTSS has negative effect on lubricin. Degradation of rhPRG4 (recombinant human lubricin) under reducing and non-reducing conditions demonstrated that CTSS is able to enzymatically cleave both monomeric and multimeric forms of lubricin. CTSS was also shown to enzymatically digest both endogenous and exogenous lubricin within human tears, abolishing its ocular surface lubricating ability and demonstrating a critical loss of function resulting from degradation by the enzyme. Their results suggested that in vivo elevated levels of CTSS in the human eye could contribute to damage and/or wear of the ocular surface.

In conclusion, lubricin is also an essential element in low-friction-force soft tissue lubrication, commonly experienced in dry-eye-related health issues and contact lens usage. Understanding its role and function can help design much more effective eye drops as well as contact lens materials. There is still much to be done, however. Transformation of this new knowledge and observations into synthetic 
systems has already emerged in the form of polymer-peptide coatings to interact with HA, as will be demonstrated in the following section.

(a)

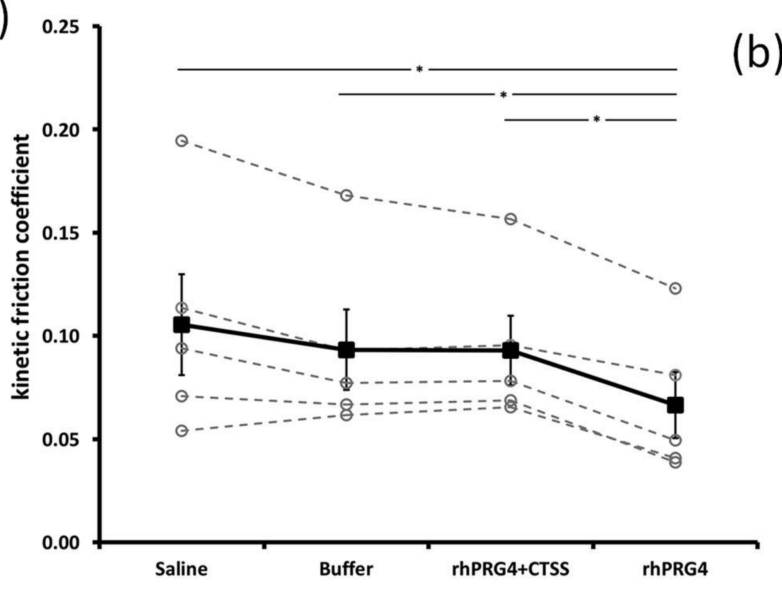

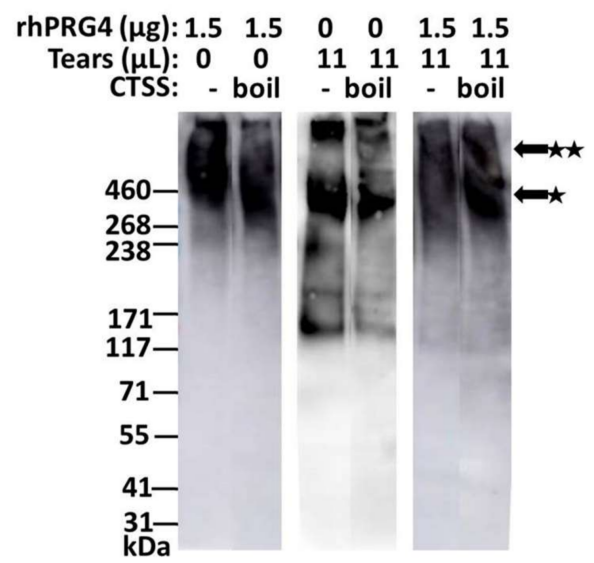

Figure 14. (a) Kinetic friction coefficient at a human eyelid - cornea interface of rhPRG4 digested by $\pm 3 \%$ CTSS under non-reducing conditions compared to saline and buffer controls. Individual data points are shown for the $n=5$ individual tests (grey), as well as the mean (black line). ${ }^{*}$ denotes statistical significance $(p<0.05)$. (b) SDS-PAGE-Western blotting of samples incubated without or $135 \mathrm{ng}$ boiled CTSS, and probed with anti-PRG4 mAb 4D6. No difference in the high MW immunoreactive PRG4 bands, monomer $(\star)$ and dimer $(\star \star)$, was observed between the sham group and samples treated with boiled (inactive) CTSS. This data confirms the observed digestion is due to the addition of active CTSS [44]. Copyright from Elsevier, 2017.

\section{New Materials and Modified Lubricin Structures for Low Friction}

Surface-grafted polymer brushes are now regarded as excellent boundary lubricants [45-47]. A very important review on this subject by Mocny and Klok [48] brings together tribological properties of surface-attached synthetic polymers with other considerations such as tribological tools of characterization and the lubrication properties of three common surface-attached polymer brushes, namely, hydrophilic, hydrophobic, and fluorinated brushes. The readers should also refer to another review by Yang and Zhou [49] on the applications of polymer brushes for antibiofouling and lubrication. Another review paper by Krishnamoorthy et al. [50] discusses advances in surface-initiated polymer brushes for biomedical applications including membrane science, bio-sensing, cell culture, antibacterial coatings, and regenerative medicine.

Samaroo et al. [51] produced lubricin-like synthetic copolymers bound to gold-coated surfaces to characterize the relationship between the polymers' molecular architecture and their lubricating capacity. They synthesized a library of nine copolymer brushes (Figure 15a) using different sizes of polyacrylic acid (PAA) and polyethylene glycol (PEG). Note that PAA formed the backbone of the polymer and PEG was the side chain. Larger-molecular-weight polymers created smoother, more densely covered surfaces. Additionally, the hydrodynamic sizes of the polymers in solution were correlated with their lubricating abilities. Friction coefficients of cartilage against polymer-treated gold surfaces were lower than cartilage against untreated surfaces. By mimicking the hydrophilic, brush-like structure of lubricin, pAA-g-PEG copolymers bound to gold surfaces were effective boundary lubricants of articular cartilage. Using different pAA backbone sizes and PEG side chain sizes, the lubricin-mimetics were tuned to create surfaces of different characteristics and different lubricating abilities (Figure 15b). In this system, pAA-g-PEG polymers were bound to gold surfaces by self-assembly via a terminal thiol. In this system, polymer size in solution correlated with lubricating ability. Notably, this relationship was not true when polymers were bound directly to cartilage [51], 
where some smaller polymers lubricated effectively. Collectively, these data point to the importance of understanding polymer lubricant conformation on both ideal surfaces and cartilage tissue.
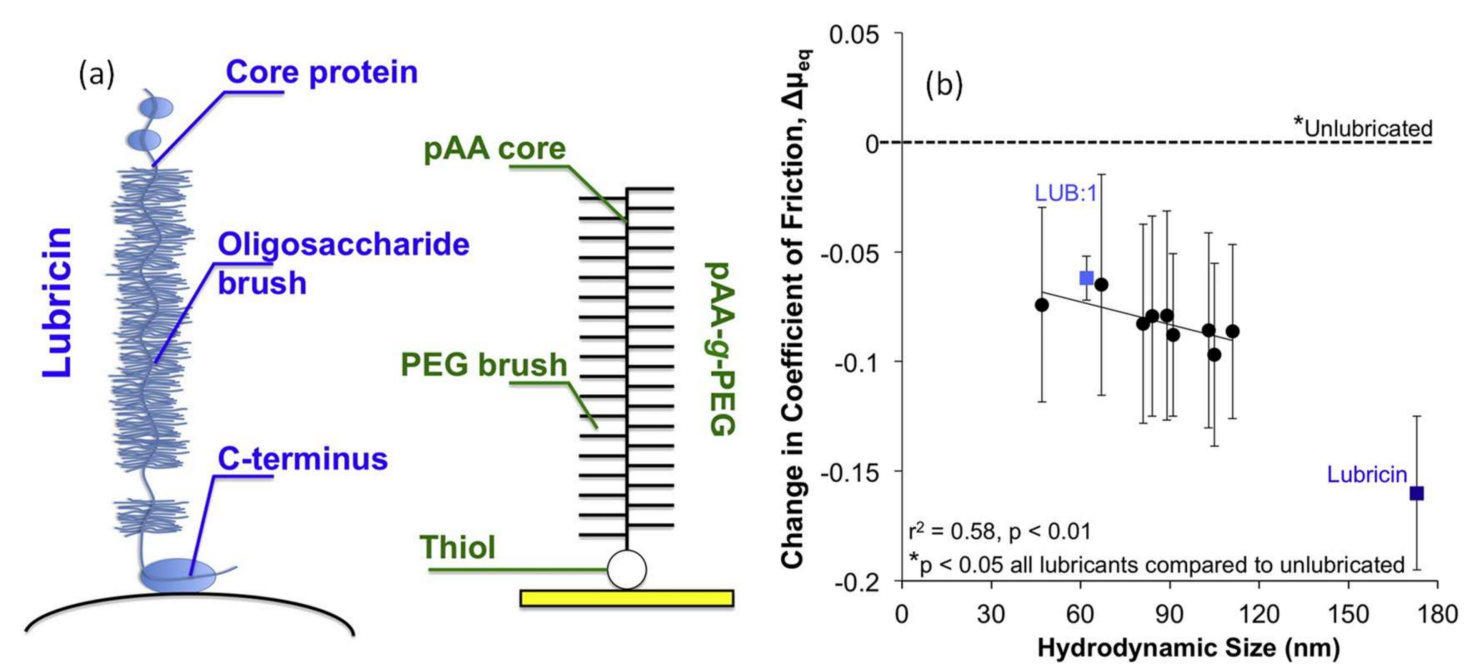

Figure 15. (a) Schematics of lubricin (left) and the pAA-g-PEG mimetic (right) are shown. Both configurations have a hydrophilic brush domain and an end terminus that anchors the molecules to surfaces. (b) The coefficients of friction of all four polymers represented in (a) were significantly lower than that of the unlubricated case. For each data point, sample sizes were $n=5$, and error bars represent standard deviation [51]. Copyright from Elsevier, 2017.

De Beer et al. [52] reported molecular dynamics simulations, as shown in Figure 16, revealing that immiscible brush systems could form slick interfaces, in which inter-digitation was eliminated and dissipation strongly reduced. Their findings were confirmed with friction force microscopy on both symmetric and asymmetric setups treated with hydrophilic and hydrophobic brush systems. In the symmetric setup, both brushes were chemically alike, while the asymmetric system consisted of two different solvent-specific brushes. The measured force trace behavior shown in Figure 16c, d and the reduction in friction were similar to the simulations and could be extended to fully immersed contacts. They postulated that two immiscible brush films in mechanical contact slide with a fluid-fluid interface with load-bearing ability, which is an ideal system for tribological applications.

Andresen Eguiluz et al. [53] performed experiments with fibronectin (FN) and its combinations with lubricin and other proteins. Fibronectin (FN) is a glycoprotein specific to the superficial zone of cartilage. Its function in lubrication and the defense against wear of articular joints is not clear. They investigated several molecular interactions among FN and other synovial fluid components such as lubricin, hyaluronan (HA), and serum albumin (SA) that are known to collectively take part in joint lubrication. Using a surface force measuring apparatus, they measured adhesion/repulsion (normal) and lateral (friction) forces throughout individual synovial fluid components that are physisorbed on FN-coated mica substrates. They concluded that (i) FN binds LUB and HA, which causes long-range repulsive normal interactions between surfaces, and that (ii) FN and lubricin synergistically augment wear resistance during shear. This is indicated by the structural integrity of FN/lubricin layers under pressures close to $4 \mathrm{MPa}$. Their findings offer new insights into the role of FN in synergistic lubrication in synovial fluid by various components under shear. This can be considered as a significant step forward in our understanding and treatment of cartilage damage due to osteoarthritis. Perry et al. [54] investigated the tribological characteristics of poly(L-lysine)-graft-poly(ethylene glycol) (PLL-g-PEG)-coated oxide interfaces using atomic force microscopy (AFM) with relation to the molecular structure. They produced polymer-bearing surfaces by spontaneous adsorption of the polymer onto the oxide substrate from a buffered solution of physiological $\mathrm{pH}$. Frictional properties of their PLL-g-PEG-coated surfaces were shown to be dependent on the deposition time and molecular construct of PLL-g-PEG as shown in 
Figure 17. To this end, they showed that the PEG chain length and the grafting ratio (i.e., the molar ratio of L-lysine monomer to PEG side chain) of adsorbed PLL-g-PEG considerably affect the frictional performance. The measured friction was reduced as the PEG chain length was increased and as the molar ratio of L-lysine monomer to PEG side chain was decreased. Specifically, their results demonstrated the need for tailoring the local conformation of brush side chains while simultaneously maintaining the backbone functionality for efficient adsorption at interfaces. Das et al. [55] showed that chemically grafted HA along with lubricin not only forms enhanced surface wear resistance but also helps reduce the friction coefficient. In particular, their work focuses on the frictional and wear behavior of some model surfaces (e.g., biopolymer coated/uncoated mica). The boundary lubrication of the surfaces has also been studied, and the role of lubricin and HA (polyelectrolyte) during the initiation and progression and later stages of wear has been investigated in their experiments. They argued that surface immobilization of HA is necessary for lubricin to function correctly with HA and to impart efficient lubricating properties to the surface. Their findings are displayed in Figure 18. The concentration of lubricin does not take part in reducing the friction for the physisorbed lubricin-HA material. They found no correlation between the friction coefficients and wear resistance under physisorption. In all cases, damage occurred at loads even less than $1 \mathrm{mN}$ regardless of the amount of lubricin. They argued that this indicated that the molecules present in the bulk play little or no role in protecting the surfaces from damage.
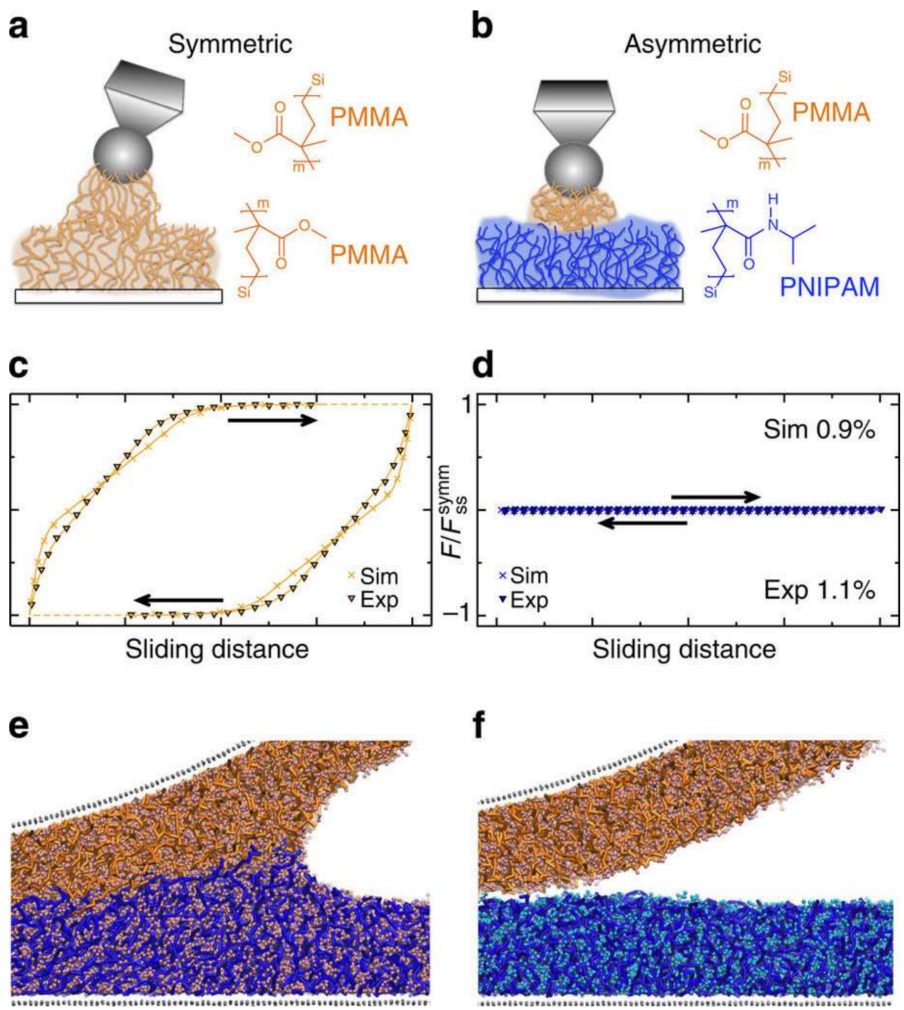

Figure 16. Schematic representation of the chemical structure and the experimental methods for symmetric (a) and asymmetric (b) contacts. Friction traces obtained from experimental (triangles) and simulated (crosses) are shown in (c,d) for the symmetric and the asymmetric cases, respectively. Friction forces $\mathrm{F}$ are normalized with steady-state friction values. The solid lines are shape function fits that depend only on two non-dimensional numbers. Simulation cell snapshots are displayed in parts (e,f). In (f), polymers with different brushes are indicated with different colors (blue) and (orange), also for the symmetric case, to visualize brush overlap. Solvent particles in the symmetric case are single-colored, and in the asymmetric system they are two-colored [52]. Copyright from Springer-Nature, 2014. 

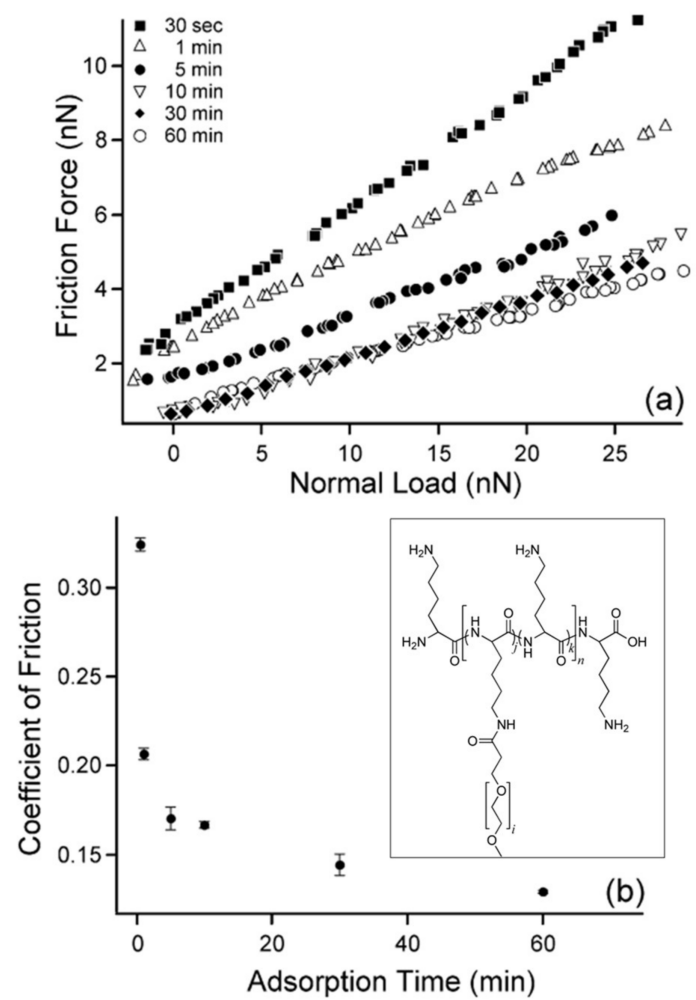

Figure 17. (a) Friction measurements as a function of applied load of a pure sodium borosilicate microsphere against PLL(20)-g[3.5]-PEG(2)-coated $\mathrm{SiO}_{2}$ substrates prepared at different polymer deposition times. (b) Plot of friction coefficient (slope of the plots in (a)) as a function of the duration of polymer deposition. The inset shows the schematic structure of a PLL-g-PEG copolymer consisting of a PLL backbone and randomly grafted PEG side chains. The designation PLL(x)-g[y]-PEG(z) indicates that the copolymer consists of a PLL backbone of molecular weight $x \mathrm{kDa}$ and a grafted PEG side chain of molecular weight $\mathrm{z}$ kDa with a grafting ratio [(lysine-mers)/(PEG side chains)] of $\mathrm{y}$ [54]. Copyright from ACS Publications, 2009.

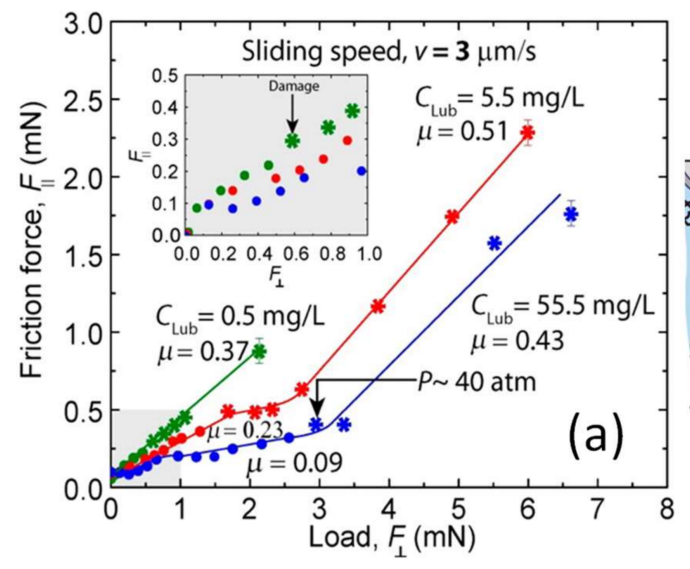

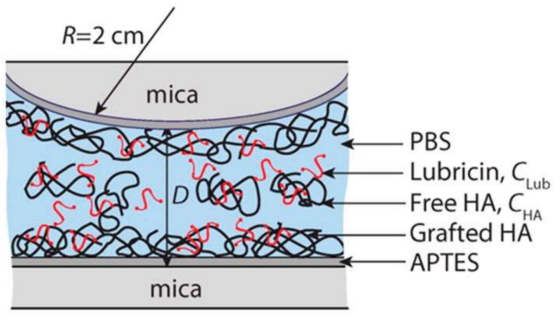

(b)

Figure 18. (a) Friction forces $\mathrm{F}_{\|}$measured as a function of the normal force (load) $\mathrm{F}_{\perp}$ between two chemically grafted HA on mica surfaces in PBS buffer with free HA $\left(\mathrm{C}_{\mathrm{HA}}=0.5-1.5 \mathrm{~g} / \mathrm{L}\right)$ and lubricin in the solution at various concentrations. The surfaces were sheared at a sliding velocity of $v=3 \mu \mathrm{m} / \mathrm{s}$. The starred points $\left(^{*}\right)$ indicate friction measurements after the surfaces became damaged. The inset shows the friction forces at the low load regime $\left(\mathrm{F}_{\perp}<1 \mathrm{mN}\right)$. (b) Schematic of the measurement system and state of the molecules used 3-aminopropyl triethoxy silane (APTES) [55]. Copyright from ACS Publications, 2013. 
In another paper, Singh et al. [56] presented a polymer-peptide-based surface coating to bind HA, which is part of synovial fluid, non-covalently to surfaces. Various tissue surfaces coated with the bound HA displayed highly encouraging lubrication properties. During in vivo tests, the coated surfaces could retain HA in the articular joint adhered to ocular tissue surfaces. They stated that such concentrated HA coatings could be physically and biologically beneficial while treating tissue-lubricating dysfunctions and could potentially be used to coat medical devices. To develop this biomimetic coating, HA-binding peptides (HABpep), obtained via phage display, were covalently or non-covalently attached to surfaces through a heterobifunctional poly(ethylene glycol) (PEG) chain (see Figure 19a-c). The HABpep-polymer system was found to non-covalently bind HA, which is either preexisting in the local fluid environment or provided exogenously, to the modified surface. They argued that localizing HA on the tissue surfaces via binding HABpep would have a number of benefits such as cartilage lubrication enhancement and better retention of HA in the articular joint. In fact, binding HA to the cartilage tissue mimics an important role of lubricin (Figure 16d).

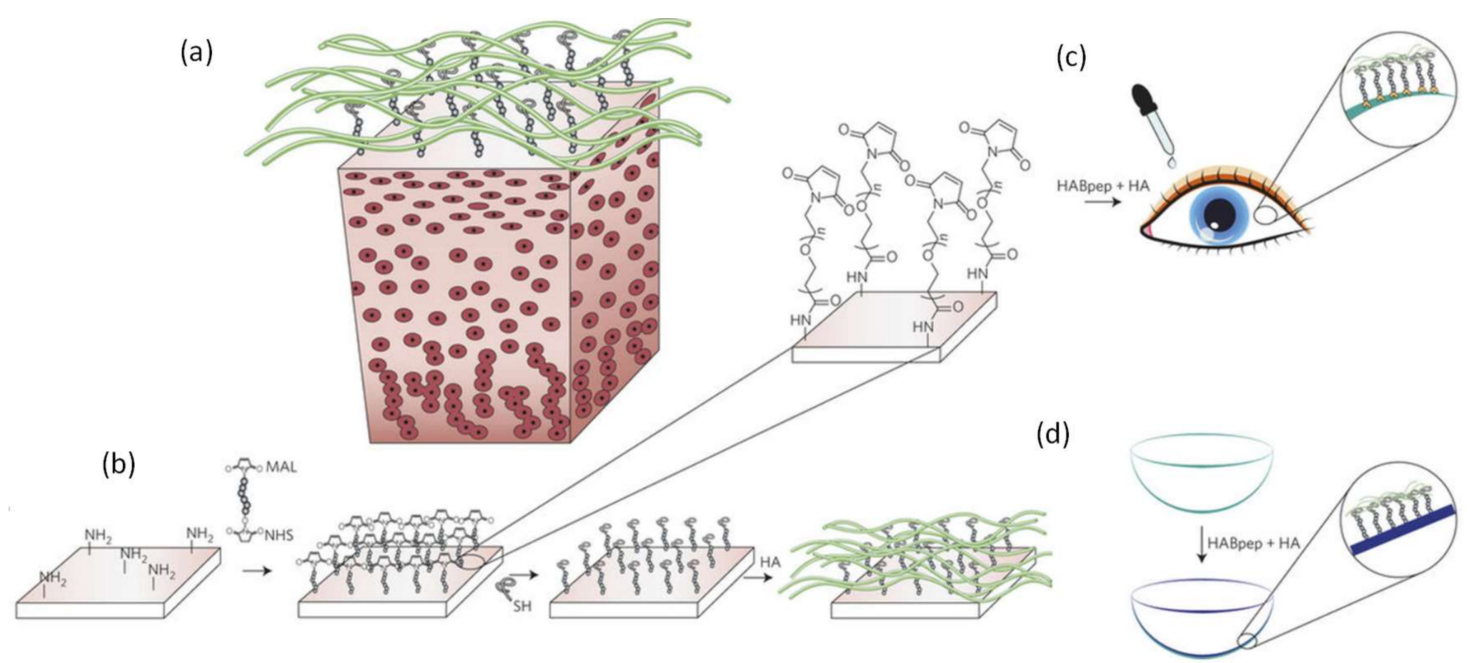

Figure 19. (a) Schematic representation of surface modified cartilage with HABpep interacting with HA in surrounding fluid. (b) An in vitro covalent cartilage surface coating process with MAL-PEG-NHS crosslinker. The primary amines of the cartilage surface create an exposed thiol-reactive surface. A thiolated HABpep is reacted with the maleimide. Upon contacting an HA solution, the HA binds to the peptide-polymer coating of the cartilage surface. (c) HABpep-polymer eye-drop solution can retain HA on the eye surface. Collagen-I-abundant eye tissues without epithelial layers, such as sclera, conjunctiva, and cornea, act as anchors for the HABpep-polymer system. (d) Contact lens is modified with the HABpep-polymer layer via the covalent reaction methodology [56]. Copyright from Springer-Nature, 2014.

Chen et al. [57] synthesized and grew polymer brushes of poly[2-(methacryloyloxy) ethyl phosphorylcholine] (pMPC) from the surfaces of macro-initiator-coated mica sheets that were attached onto cylindrical lenses for surface force balance (SFB) measurements. Their motivation was biomimicry relevant to boundary lubrication in human-made systems in aqueous or physiological media, as in biomedical devices, in which friction and wear are often an issue. They argued that, although their polyzwitterionic brushes were not analogous to cartilage surfaces, their findings suggest the possible importance at such surfaces of highly hydrated macromolecules in both chondroprotective and lubrication roles. After calibration in dry air, control measurements of the normal forces $\left(F_{\mathrm{n}}\right)$ and shear forces $\left(F_{\mathrm{S}}\right)$ between the macro-initiator-coated mica surfaces were conducted as a function of their separation and sliding velocity, respectively. They performed measurements on both in polymer-free water and after incubation in a $0.3 \% w / v$ pMPC homo-polymer solution to compare with the subsequent pMPC brush profiles (See Figure 20). 


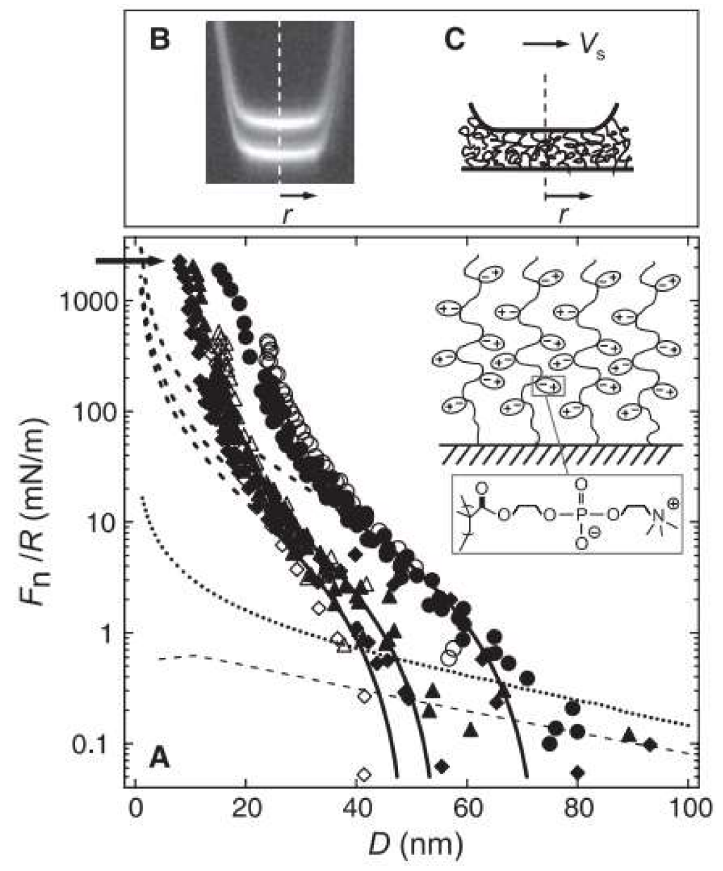

Figure 20. Results on the normal interactions between pMPC brush-coated mica surfaces in conjunction with water and aqueous salt solutions (results are normalized). (A) Normal force profiles: circles, pure water; triangles, $8.7 \mathrm{mM} \mathrm{NaNO}_{3}$ solution; diamonds, $88.6 \mathrm{mM} \mathrm{NaNO}_{3}$ solution. Solid and open symbols indicate compression and decompression, respectively. The pMPC brush-like configuration and its monomer structure are also shown. The dashed and dotted lines indicate brush-free control experiments between bare and between macroinitiator-coated mica, respectively. (B) Equal chromatic order interference fringes showing flattening of the mica surfaces under high loads, indicated by the arrow in (A). (C) The schematic configuration in (B) with contact area $A \cong \pi \mathrm{r}^{2}$ ) [57]. Copyright from American Association for the Advancement of Science (AAAS), 2009.

Liu et al. [58] utilized new multifunctional nanoparticles to achieve hydration lubrication and efficient drug delivery. The nanoparticles were core/shell charged polymer brushes containing hollow silica particles (HSNPs) grafted with poly(3-sulfopropyl methacrylate potassium salt) (PSPMA). They were synthesized by surface-initiated atom transfer radical polymerization (ATRP). HSNPs having a silica shell of about $28 \mathrm{~nm}$ were coated by a polymer brush layer of about $40 \mathrm{~nm}$. PSPMA brushes demonstrated good hydration properties, and PSPMA-brushes-grafted HSNPs could swell effectively in aqueous media. Their rheological characterization, tribological tests, and absorption analysis results suggested that the composite nanoparticles can achieve an efficient hydration lubrication effect. When a drug such as aspirin was used as a model, it could be encapsulated by the hollow nanoparticles causing a significant sustained drug release. They claimed that the combination of hydration lubrication and drug loading capability could render the core/shell charged polymer brushes-grafted hollow silica particles a promising material for joint lubrication and arthritis treatment.

Liu et al. [59] reported on the fabrication of microgels comprising poly( $N$-isopropylacrylamide) (PNIPAAm) grafted with poly(3-sulfopropyl methacrylate potassium salt) (PSPMK) brushes. They further discussed their potential use as artificial synovial fluid for biomimetic aqueous lubrication systems for arthritis treatment. In order to conduct experiments on water-based hydration lubrication and temperature-triggered drug release, they chose negatively charged PSPMK brushes and thermosensitive PNIPAAm, respectively. Under soft friction pairs (see Figure 21), an ultra-low coefficient of friction was achieved, whilst the brush-like thermosensitive microgels showed a desirable temperature-triggered drug release performance. They argued that such soft charged brush-like microgels offer a great possibility for designing intelligent synovial fluids and that the collective 
lubrication and drug-loading capabilities of these soft brush-like nanoparticles ensures potentials for large scale clinical applications as synthetic joint lubricant fluid in arthritis treatment.

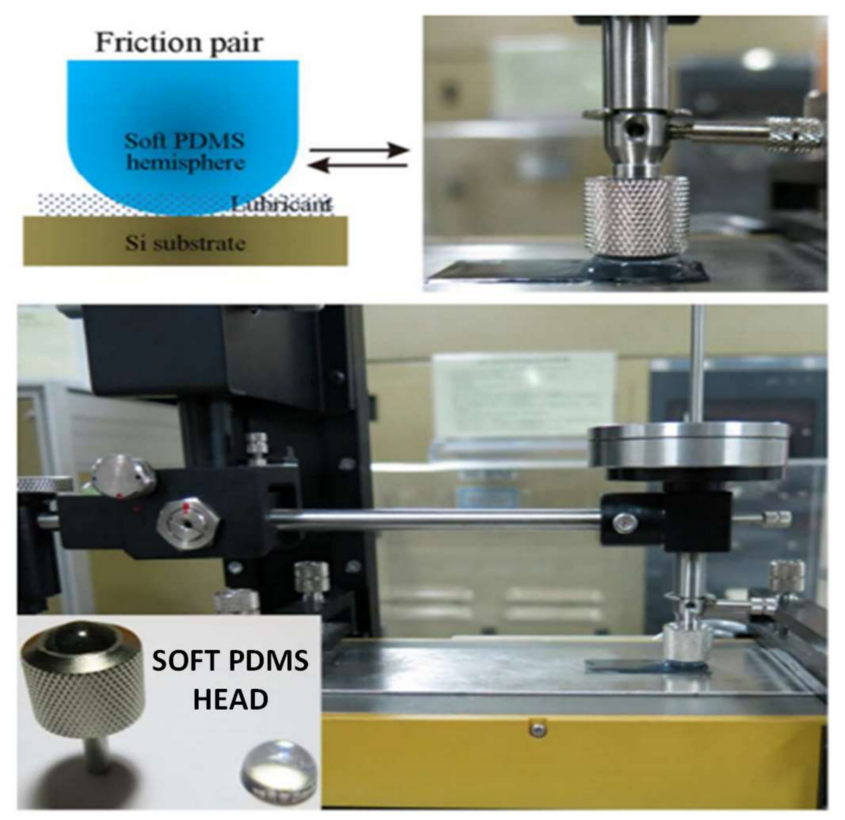

Figure 21. Schematic diagram illustrates the modified friction pairs, and the inset displays the elastic polydimethylsiloxane (PDMS) hemisphere instead of traditional steel ball [59]. Copyright from ACS Publications, 2014.

Ishihara [60] reviewed advances in the surface modification of ultra-high molecular-weight polyethylene (UHMWPE) using polymer brushes (see Figure 22). Through their work, an articular cartilage-mimicking nano-textured surface was prepared by grafting poly(2-methacryloyloxyethyl phosphorylcholine) or poly(MPC) onto a highly cross-linked UHMWPE (X-UHMWPE) by photo-initiated polymerization. The thickness of the poly(MPC) graft layer was measured to be 100-200 nm. This treatment was found to increase the surface hydrophilicity of this hydrophobic polymer.
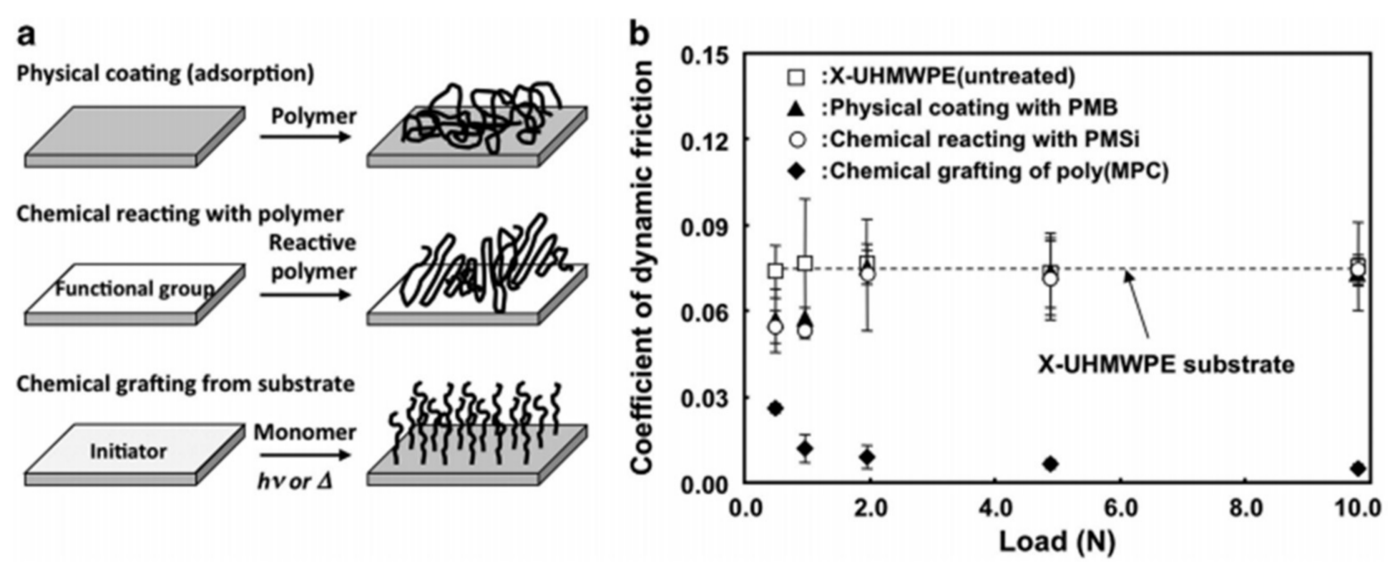

Figure 22. (a) Schematic displaying hydrophilic polymer modification processes on the substrate. (b) Dynamic friction coefficient of the untreated cross-linked ultra-high-molecular-weight polyethylene (X-UHMWPE), physical (no chemical reaction) coating with poly(MPC)-co- $n$-butyl methacrylate (PMB), chemical reaction with poly(MPC-co-3-methacryloyloxypropyl trimethoxysilane) (PMSi), and chemical grafting of poly(MPC) on X-UHMWPE as a function of applied ball-on-plate load. Data are expressed as the mean \pm standard deviation [60]. Copyright from Springer-Nature, 2015. 
Nie et al. [61] reviewed a number of nanocomposites fabricated by embedding inorganic nanoparticles (NPs) in polymer brushes to function as tribo-surfaces. They showed that polymer brushes are predominantly convenient media for the preparation of nanocomposites, as the macromolecular matrix functions as a reaction chamber for nanoparticle synthesis, as a scaffold for immobilization, and as a capping agent to prevent nanoparticle aggregation. Moreover, they argued that synergies between the polymer chains and the inorganic NPs would grant the composite new properties such as in sensors, detectors, and electronic/optical devices. They reviewed a number of polymer brush systems (e.g., polyelectrolytes and polyampholytes) for the incorporation of inorganic NPs to make composite surfaces/NPs with specific functions.

Raj et al. [62] suggested that cartilage oligomeric matrix protein (COMP) is one of the biomolecules that promotes anchoring of lubricin facilitating lubrication. They conducted an experimental study in which weakly hydrophobic poly(methyl methacrylate) (PMMA) surfaces were coated by COMP and lubricin, individually and in combinations (see Figure 23). Adsorption was investigated using a quartz crystal microbalance, and friction between the biopolymer-coated surfaces was determined by employing the atomic force microscope-colloidal probe technique. They reported that COMP facilitated firm, directed attachment of lubricin in a manner that resulted in low-friction forces, significantly lower than what was achieved when lubricin was directly adsorbed to PMMA.

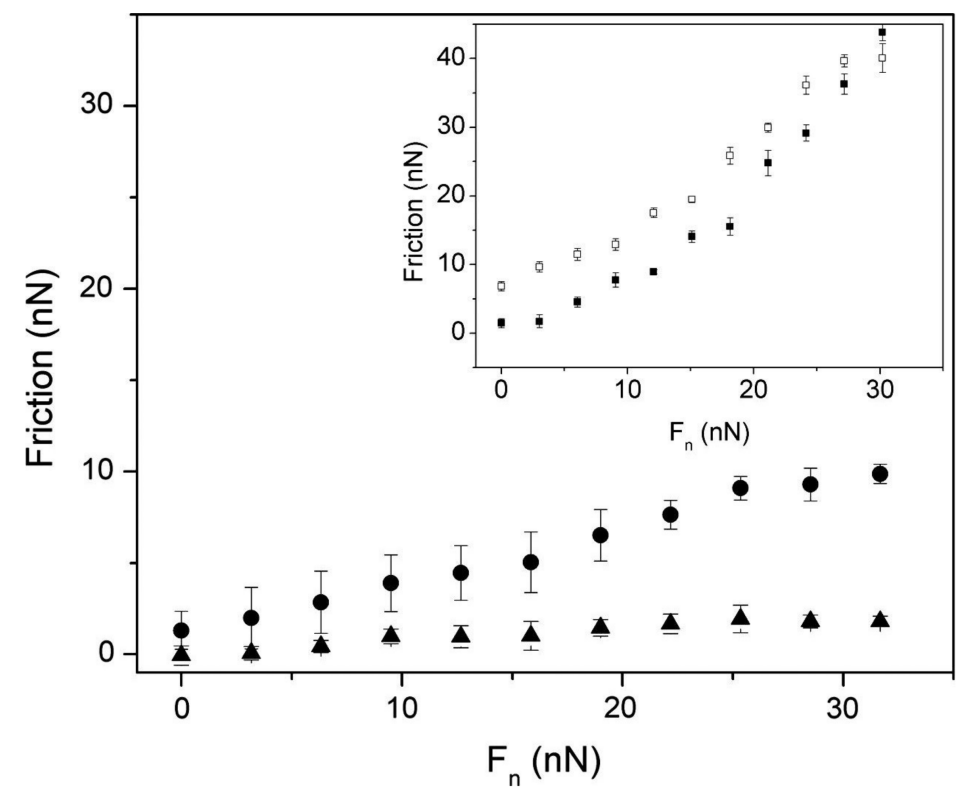

Figure 23. A plot displaying friction force as a function of applied load. The system is made up of two PMMA surfaces coated with COMP (solid circles), and COMP-lubricin (triangles). The label of the $y$-axis Friction indicates friction force, and the $x$-axis label $F_{\mathrm{n}}$ indicates applied load. Inset: Same system coated with lubricin (solid and open squares-loading and unloading, respectively) [62]. Copyright from Elsevier, 2016.

A recent paper by Suchaoin et al. [63] targeted generation and characterization of chondroitin sulfate-cysteine conjugate (CS-cys) as a novel bioadhesive agent for intra-articular use. Muco-adhesive and rheological properties were investigated, while bioadhesive features of CS-cys on porcine articular cartilage were evaluated via tensile studies. Thiol groups were introduced to chondroitin sulfate (CS) in order to improve bio-adhesion. Thiol group addition caused an increase in dynamic viscosity of CS-cys as a function of time. Moreover, improved adhesion of CS-cys was found to be due to disulfide crosslinking between thiomers and cysteine-rich domains of biological glycoprotein surfaces. They claimed that this improved bio-adhesion property of CS-cys can be utilized as an intra-articular agent for treatment of osteoarthritis. 
Chang et al. [64] used self-assembled monolayer functionalized model substrates having hydroxyl or methyl terminating groups to study the effect of surface chemistry on lubricin and HA adsorption. They used surface plasmon resonance (SPR) to study normal force interactions between these surfaces treated with different lubricin and HA concentrations using colloidal probe microscopy. They found that lubricin is amphiphilic and can readily adsorb onto both methyl- and hydroxyl-terminated surfaces.

On hydrophobic surfaces, lubricin conformed to a compact, loop-like shape where its hydrophobic domains at the $\mathrm{N}$ and $\mathrm{C}$ termini helped anchor to surfaces. On hydrophilic surfaces, lubricin easily adsorbed through its hydrophilic central domain and conformed to an extended tail-like conformation with increasing solution concentration (see Figure 24). They further demonstrated that lubricin developed strong repulsive interactions when two surfaces were brought into contact and after separation of surfaces; adhesion occurred as a result of molecular bridging and chain disentanglement. For a mixture of lubricin and HA, they reported somewhat more adsorptions and repulsions compared to the measurements of lubricin alone. They concluded that the ability of lubricin to adhere to different surfaces while conforming to loop and tail-like layer structures due to overlap can result in strong repulsive interactions. This can enable lubricin to act as an efficient antiadhesive and chondroprotectant on cartilage surfaces, hence preventing or minimizing wear.

a) $\mathrm{CH}_{3}$, low concentration

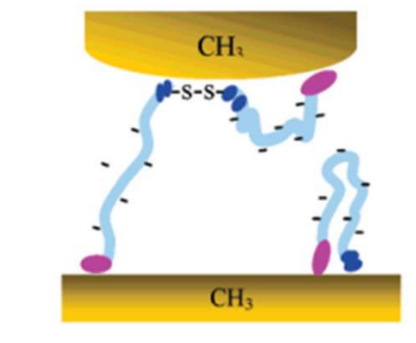

c) $\mathrm{CH}_{3}$, high concentration

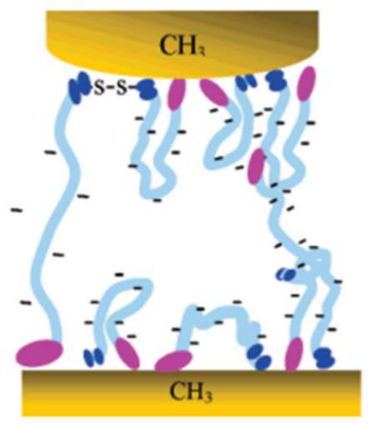

b) $\mathrm{OH}$, low concentration

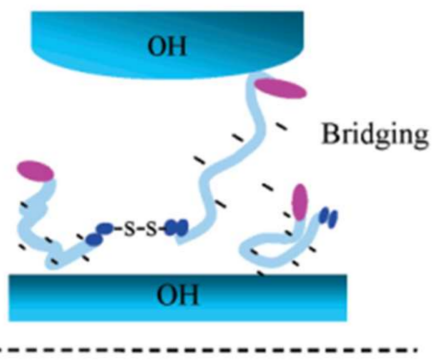

d) $\mathrm{OH}$, high concentration

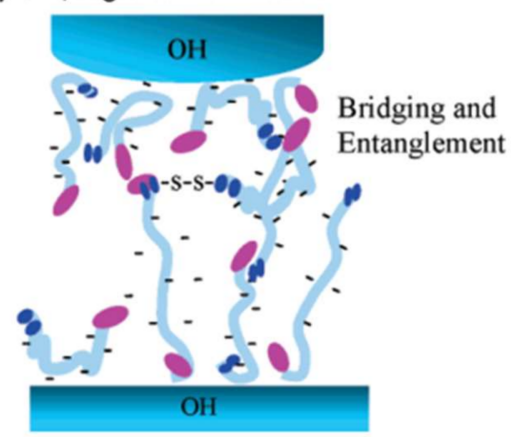

Figure 24. Schematic representation of the steric and bridging interactions of lubricin on hydrophobic, methyl-terminated $(\mathbf{a}, \mathbf{c})$, and hydrophilic, hydroxyl-terminated $(\mathbf{b}, \mathbf{d})$ SAM surfaces at low $(\mathbf{a}, \mathbf{b})$ and high surface coverage (c,d) [64]. Copyright from ACS Publications, 2008.

Andresen Eguiluz et al. [65] used a co-polymer polyacrylic acid-co-polyethylene glycol to simulate lubricin and investigated its interaction with fibronectin on mica surfaces. They labeled the polymer as mimLUB, and focused on friction and wear mediation between synthetic mimLUB-coated mica surfaces upon shear, at the molecular scale. They reported that the presence of fibronectin was essential to link mimLUB to either cartilage or implant surfaces for enhancing wear protection during prolonged shear up to contact pressures of 3.4 MPa. This effect was attributed to fibronectin-mediated strong anchoring, hence preventing removal of polymer brush structures under high-pressure shear forces. They stressed that synergistic lubrication mechanisms of multicomponent systems could be 
a effective means to treat damaged cartilage surfaces through the fibronectin layer instead of directly using lubricin.

\section{Conclusions and Outlook}

Although both lubricin and HA are important molecular constituents of synovial fluid, recent biomedical research findings indicate that lubricin plays a more important role in boundary lubrication, including soft tissues such as eyelids [66]. Many different physiological evidences show that decrease in synovial lubricin concentrations following ageing or injury can cause moderate or severe wear-induced damage and related deterioration in boundary lubrication, potentially leading to osteoarthritis [67]. Transformation of this knowledge into polymer science and tribology leads to the design of new bioinspired lubricants. As such, lubricin-inspired research is currently developing nanostructured polymers as new materials not only for the treatment of chronic diseases such as osteoarthritis or multiple sclerosis but also for machine lubrication. The main scientific approach consists of characterizing the interaction of such polymers with biological interfaces at the nanoscale to optimize their properties. Understanding lubricin can lead to the development of synthetic cartilage that can be used to treat damaged areas and new synovial fluid as a supplementary fluid using biocompatible polymers. To reach these goals, researchers need to mimic complex lubricin interactions and morphology into polymeric structures and test them in terms of nano-tribology and toxicity. This would further lead to the formulation of nano-structured hydrogels composed of an interpenetrated network allowing strong mechanical resistance and controlled synovial fluid release under load and shear.

It is important to note that these biological lubricants that are present in synovial fluids experience pressures of the order of $100 \mathrm{~atm}$ [68]. This once again shows how important it is to understand these mechanisms well and to adapt them to real engineering tribology. This review attempted to demonstrate recent progress in the investigation of lubricin and lubricin-inspired materials and to summarize their general design principles (i.e., the interplay between chemical constituents and morphological features). Following natural design principles, several artificial low-friction materials have shown unique and superior tribological properties. This review has also established that recent synthetic assembling technologies are still far from reproducing sophisticated lubricin structures and dynamics, whose dimensions span from nanoscale to macroscale. However, with the help of new characterization technologies and manufacturing methods, significant progress has been made toward designing low-friction soft materials. It is believed that bio-based abrasion and/or wear resistant materials will have much more promising future applications as new nature- and bio-inspired procedures merge with advanced engineering technologies.

An important aspect that is generally overlooked is the fact that synovial fluid is a viscoelastic polymeric solution [69]. Despite this apparent viscoelastic nature of synovial fluid, many approach synovial fluid as a viscous fluid. This is attributed to the fact that the synovial fluid behaves as a generalized viscous fluid so long as it does not experience instantaneous external stimuli. In other words, under steady-state conditions with no instantaneous components, the fluid response is that of a generalized viscous fluid [69]. Mimicking lubricin for industrial tribology could be beneficial once new ways to design synovial-like fluids containing nano-additives could be developed, for instance, for industrial pipe or pump flow systems [70]. Also, during medical treatments, natural therapeutic polymers/proteins that can be injected into damaged joints that are less susceptible to enzymatic degradation and bind to the articular surface to reduce friction can cause full healing of early osteoarthritis [71]. Therefore, new lubricin-like molecules should be searched and synthesized and, along with other published relevant materials, should be subjected to preclinical investigations, for example, in cases immediately following an injury to the joint, as a protective measure with regard to preventing osteoarthritis pathogenesis.

Conflicts of Interest: The author declares no conflict of interest. 


\section{References}

1. Dèdinaitè, A. Biomimetic lubrication. Soft Matter 2012, 8, 273-284. [CrossRef]

2. Dėdinaitè, A.; Claesson, P.M. Synergies in lubrication. Phys. Chem. Chem. Phys. 2017, 19, 23677-23689. [CrossRef] [PubMed]

3. Divandari, M.; Morgese, G.; Trachsel, L.; Romio, M.; Dehghani, E.S.; Rosenboom, J.G.; Paradisi, C.; Zenobi-Wong, M.; Ramakrishna, S.N.; Benetti, E.M. Topology Effects on the Structural and Physicochemical Properties of Polymer Brushes. Macromolecules 2017, 50, 7760-7769. [CrossRef]

4. Jay, G.D.; Torres, J.R.; Rhee, D.K.; Helminen, H.J.; Hytinnen, M.M.; Cha, C.J.; Warman, M.L. Association between friction and wear in diarthrodial joints lacking lubricin. Arthritis Rheumatol. 2007, 56, 3662-3669. [CrossRef] [PubMed]

5. Bonnevie, E.D.; Galesso, D.; Secchieri, C.; Cohen, I.; Bonassar, L.J. Elastoviscous transitions of articular cartilage reveal a mechanism of synergy between lubricin and hyaluronic acid. PLoS ONE 2015, 10, e0143415. [CrossRef] [PubMed]

6. Siddaiah, A.; Menezes, P.L. Advances in Bio-inspired Tribology for Engineering Applications. J. BioTribo-Corros. 2016, 2, 23. [CrossRef]

7. Meng, J.; Zhang, P.; Wang, S. Recent progress of abrasion-resistant materials: Learning from nature. Chem. Soc. Rev. 2016, 45, 237-251. [CrossRef] [PubMed]

8. Gebeshuber, I.C.; Drack, M.; Scherge, M. Tribology in biology. Tribology-Materials. Surf. Interfaces 2008, 2, 200-212. [CrossRef]

9. McKellop, H.; Clarke, I.; Markolf, K.; Amstutz, H. Friction and wear properties of polymer, metal, and ceramic prosthetic joint materials evaluated on a multichannel screening device. J. Biomed. Mater. Res. Part A 1981, 15, 619-653. [CrossRef] [PubMed]

10. Hauert, R. A review of modified DLC coatings for biological applications. Diam. Relat. Mater. 2003, 12, 583-589. [CrossRef]

11. Blum, M.M.; Ovaert, T.C. Investigation of friction and surface degradation of innovative boundary lubricant functionalized hydrogel material for use as artificial articular cartilage. Wear 2013, 301, 201-209. [CrossRef]

12. Hoseini, M.; Jedenmalm, A.; Boldizar, A. Tribological investigation of coatings for artificial joints. Wear 2008, 264, 958-966. [CrossRef]

13. Jay, G.D.; Waller, K.A. The biology of lubricin: Near frictionless joint motion. Matrix Biol. 2014, 39, 17-24. [CrossRef] [PubMed]

14. Musumeci, G. The role of lubricin in normal and pathological joint tissue: A contemporary review. OA Anat. 2013, 1, 2. [CrossRef]

15. Samaroo, K.J.; Tan, M.; Putnam, D.; Bonassar, L.J. Binding and lubrication of biomimetic boundary lubricants on articular cartilage. J. Orthop. Res. 2017, 35, 548-557. [CrossRef] [PubMed]

16. Grupp, T.M.; Utzschneider, S.; Schröder, C.; Schwiesau, J.; Fritz, B.; Maas, A.; Jansson, V. Biotribology of alternative bearing materials for unicompartmental knee arthroplasty. Acta Biomater. 2010, 6, 3601-3610. [CrossRef] [PubMed]

17. Gebeshuber, I.C. Biotribology inspires new technologies. Nano Today 2007, 2, 30-37. [CrossRef]

18. Zhou, Z.R.; Jin, Z.M. Biotribology: Recent progresses and future perspectives. Biosurf. Biotribol. 2015, 1, 3-24. [CrossRef]

19. Grupp, T.M.; Holderied, M.; Mulliez, M.A.; Streller, R.; Jäger, M.; Blömer, W.; Utzschneider, S. Biotribology of a vitamin E-stabilized polyethylene for hip arthroplasty-Influence of artificial ageing and third-body particles on wear. Acta Biomater. 2014, 10, 3068-3078. [CrossRef] [PubMed]

20. Freeman, M.E.; Furey, M.J.; Love, B.J.; Hampton, J.M. Friction, wear, and lubrication of hydrogels as synthetic articular cartilage. Wear 2000, 241, 129-135. [CrossRef]

21. Biswas, S.K.; Vijayan, K. Friction and wear of PTFE-A review. Wear 1992, 158, 193-211. [CrossRef]

22. Trunfio-Sfarghiu, A.M.; Berthier, Y.; Meurisse, M.H.; Rieu, J.P. Role of nanomechanical properties in the tribological performance of phospholipid biomimetic surfaces. Langmuir, 2008, 24, 8765-8771. [CrossRef] [PubMed]

23. Askary, A.; Smeeton, J.; Paul, S.; Schindler, S.; Braasch, I.; Ellis, N.A.; Crump, J.G. Ancient origin of lubricated joints in bony vertebrates. eLife 2016, 5, e16415. [CrossRef] [PubMed] 
24. Boettcher, K.; Winkeljann, B.; Schmidt, T.A.; Lieleg, O. Quantification of cartilage wear morphologies in unidirectional sliding experiments: Influence of different macromolecular lubricants. Biotribology 2017, 12, 43-51. [CrossRef]

25. Zappone, B.; Ruths, M.; Greene, G.W.; Jay, G.D.; Israelachvili, J.N. Adsorption, lubrication, and wear of lubricin on model surfaces: Polymer brush-like behavior of a glycoprotein. Biophys. J. 2007, 92, 1693-1708. [CrossRef] [PubMed]

26. Banquy, X.; Burdyńska, J.; Lee, D.W.; Matyjaszewski, K.; Israelachvili, J. Bioinspired bottlebrush polymer exhibits low friction and Amontons-like behavior. J. Am. Chem. Soc. 2014, 136, 6199-6202. [CrossRef] [PubMed]

27. Xuan, S.; Gupta, S.; Li, X.; Bleuel, M.; Schneider, G.J.; Zhang, D. Synthesis and Characterization of Well-Defined PEGylated Polypeptoids as Protein-Resistant Polymers. Biomacromolecules 2017, 18, 951-964. [CrossRef] [PubMed]

28. Szychlinska, M.A.; Trovato, F.M.; Di Rosa, M.; Malaguarnera, L.; Puzzo, L.; Leonardi, R.; Musumeci, G. Co-expression and co-localization of cartilage glycoproteins CHI3L1 and lubricin in osteoarthritic cartilage: Morphological, immunohistochemical and gene expression profiles. Int. J. Mol. Sci. 2016, 17, 359. [CrossRef] [PubMed]

29. Aninwene, G.E.; Yang, Z.; Ravi, V.; Jay, G.D.; Webster, T.J. Lubricin as a novel nanostructured protein coating to reduce fibroblast density. Int. J. Nanomed. 2014, 9, 3131-3135.

30. Iqbal, S.M.; Leonard, C.; Regmi, S.C.; De Rantere, D.; Tailor, P.; Ren, G.; Salo, P.T. Lubricin/proteoglycan 4 binds to and regulates the activity of toll-like receptors in vitro. Sci. Rep. 2016, 6, 18910. [CrossRef] [PubMed]

31. Rhee, D.K.; Marcelino, J.; Baker, M.; Gong, Y.; Smits, P.; Lefebvre, V.; Carpten, J.D. The secreted glycoprotein lubricin protects cartilage surfaces and inhibits synovial cell overgrowth. J. Clin. Investig. 2005, 115, 622-631. [CrossRef] [PubMed]

32. Jones, A.R.; Gleghorn, J.P.; Hughes, C.E.; Fitz, L.J.; Zollner, R.; Wainwright, S.D.; Flannery, C.R. Binding and localization of recombinant lubricin to articular cartilage surfaces. J. Orthop. Res. 2007, 25, $283-292$. [CrossRef] [PubMed]

33. Hussainova, I.; Ghaemi, H. Biobearings: Where mechanics meets biology. Proc. Estonian Acad. Sci. 2008, 57, 232-240. [CrossRef]

34. Xu, Y.; Zhan, J.M.; Zheng, Y.H.; Han, Y.; Zhang, Z.G.; Xi, C. Computational synovial dynamics of a normal temporomandibular joint during jaw opening. J. Formos. Med. Assoc. 2013, 112, 346-351. [CrossRef] [PubMed]

35. Waller, K.A.; Zhang, L.X.; Elsaid, K.A.; Fleming, B.C.; Warman, M.L.; Jay, G.D. Role of lubricin and boundary lubrication in the prevention of chondrocyte apoptosis. Proc. Natl. Acad. Sci. USA 2013, 110, 5852-5857. [CrossRef] [PubMed]

36. Wong, B.L.; Kim, S.C.; Antonacci, J.M.; McIlwraith, C.W.; Sah, R.L. Cartilage shear dynamics during tibio-femoral articulation: Effect of acute joint injury and tribosupplementation on synovial fluid lubrication. Osteoarthr. Cartil. 2010, 18, 464-471. [CrossRef] [PubMed]

37. Reesink, H.L.; Bonnevie, E.D.; Liu, S.; Shurer, C.R.; Hollander, M.J.; Bonassar, L.J.; Nixon, A.J. Galectin-3 binds to lubricin and reinforces the lubricating boundary layer of articular cartilage. Sci. Rep. 2016, 6, 25463. [CrossRef] [PubMed]

38. Samsom, M.L.; Morrison, S.; Masala, N.; Sullivan, B.D.; Sullivan, D.A.; Sheardown, H.; Schmidt, T.A. Characterization of full-length recombinant human proteoglycan 4 as an ocular surface boundary lubricant. Exp. Eye Res. 2014, 127, 14-19. [CrossRef] [PubMed]

39. Lambiase, A.; Sullivan, B.D.; Schmidt, T.A.; Sullivan, D.A.; Jay, G.D.; Truitt, E.R.; Mantelli, F. A two-week, randomized, double-masked study to evaluate safety and efficacy of lubricin $(150 \mu \mathrm{g} / \mathrm{mL})$ eye drops versus sodium hyaluronate (HA) $0.18 \%$ eye drops $\left(\right.$ Vismed $^{\circledR}$ ) in patients with moderate dry eye disease. Ocul. Surf. 2017, 15, 77-87. [CrossRef] [PubMed]

40. Samsom, M.; Iwabuchi, Y.; Sheardown, H.; Schmidt, T.A. Proteoglycan 4 and hyaluronan as boundary lubricants for model contact lens hydrogels. J. Biomed. Mater. Res. Part B 2017, 106, 1329-1338. [CrossRef] [PubMed]

41. Morrison, S.; Sullivan, D.A.; Sullivan, B.D.; Sheardown, H.; Schmidt, T.A. Dose-dependent and synergistic effects of proteoglycan 4 on boundary lubrication at a human cornea-polydimethylsiloxane biointerface. Eye Contact Lens 2012, 38, 27-35. [CrossRef] [PubMed] 
42. Samsom, M.; Chan, A.; Iwabuchi, Y.; Subbaraman, L.; Jones, L.; Schmidt, T.A. In vitro friction testing of contact lenses and human ocular tissues: Effect of proteoglycan 4 (PRG4). Tribol. Int. 2015, 89, 27-33. [CrossRef]

43. Schmidt, T.A.; Sullivan, D.A.; Knop, E.; Richards, S.M.; Knop, N.; Liu, S.; Sullivan, B.D. Transcription, translation, and function of lubricin, a boundary lubricant, at the ocular surface. JAMA Ophthalmol. 2013, 131, 766-776. [CrossRef] [PubMed]

44. Regmi, S.C.; Samsom, M.L.; Heynen, M.L.; Jay, G.D.; Sullivan, B.D.; Srinivasan, S.; Schmidt, T.A. degradation of proteoglycan 4/lubricin by cathepsin S: Potential mechanism for diminished ocular surface lubrication in Sjögren's syndrome. Exp. Eye Res. 2017, 161, 1-9. [CrossRef] [PubMed]

45. Grest, G.S. Computer simulations of shear and friction between polymer brushes. Curr. Opin. Colloid Interface Sci. 1997, 2, 271-277. [CrossRef]

46. Kreer, T. Polymer-brush lubrication: A review of recent theoretical advances. Soft Matter 2016, 12, 3479-3501. [CrossRef] [PubMed]

47. Azzaroni, O.; Szleifer, I. Polymer and Biopolymer Brushes: For Materials Science and Biotechnology; John Wiley \& Sons: Hoboken, NJ, USA, 2017.

48. Mocny, P.; Klok, H.A. Tribology of surface-grafted polymer brushes. Mol. Syst. Des. Eng. 2016, 1, 141-154. [CrossRef]

49. Yang, W.; Zhou, F. Polymer brushes for antibiofouling and lubrication. Biosurf. Biotribol. 2017, 3, 97-114. [CrossRef]

50. Krishnamoorthy, M.; Hakobyan, S.; Ramstedt, M.; Gautrot, J.E. Surface-initiated polymer brushes in the biomedical field: Applications in membrane science, biosensing, cell culture, regenerative medicine and antibacterial coatings. Chem. Rev. 2014, 114, 10976-11026. [CrossRef] [PubMed]

51. Samaroo, K.J.; Tan, M.; Eguiluz, R.C.A.; Gourdon, D.; Putnam, D.; Bonassar, L.J. Tunable Lubricin-mimetics for Boundary Lubrication of Cartilage. Biotribology 2017, 9, 18-23. [CrossRef]

52. De Beer, S.; Kutnyanszky, E.; Schön, P.M.; Vancso, G.J.; Müser, M.H. Solvent-induced immiscibility of polymer brushes eliminates dissipation channels. Nat. Commun. 2014, 5, 3781. [CrossRef] [PubMed]

53. Andresen Eguiluz, R.C.; Cook, S.G.; Brown, C.N.; Wu, F.; Pacifici, N.J.; Bonassar, L.J.; Gourdon, D. Fibronectin mediates enhanced wear protection of lubricin during shear. Biomacromolecules 2015, 16, 2884-2894. [CrossRef] [PubMed]

54. Perry, S.S.; Yan, X.; Limpoco, F.T.; Lee, S.; Müller, M.; Spencer, N.D. Tribological properties of poly(L-lysine)graft-poly(ethylene glycol) films: Influence of polymer architecture and adsorbed conformation. ACS Appl. Mater. Interfaces 2009, 1, 1224-1230. [CrossRef] [PubMed]

55. Das, S.; Banquy, X.; Zappone, B.; Greene, G.W.; Jay, G.D.; Israelachvili, J.N. Synergistic interactions between grafted Coles and lubricin provide enhanced wear protection and lubrication. Biomacromolecules 2013, 14, 1669-1677. [CrossRef] [PubMed]

56. Singh, A.; Corvelli, M.; Unterman, S.A.; Wepasnick, K.A.; McDonnell, P.; Elisseeff, J.H. Enhanced lubrication on tissue and biomaterial surfaces through peptide-mediated binding of hyaluronic acid. Nat. Mater. 2014, 13, 988-995. [CrossRef] [PubMed]

57. Chen, M.; Briscoe, W.H.; Armes, S.P.; Klein, J. Lubrication at physiological pressures by polyzwitterionic brushes. Science 2009, 323, 1698-1701. [CrossRef] [PubMed]

58. Liu, G.; Cai, M.; Zhou, F.; Liu, W. Charged polymer brushes-grafted hollow silica nanoparticles as a novel promising material for simultaneous joint lubrication and treatment. J. Phys. Chem. B 2014, 118, 4920-4931. [CrossRef] [PubMed]

59. Liu, G.; Liu, Z.; Li, N.; Wang, X.; Zhou, F.; Liu, W. Hairy polyelectrolyte brushes-grafted thermosensitive microgels as artificial synovial fluid for simultaneous biomimetic lubrication and arthritis treatment. ACS Appl. Mater. Interfaces 2014, 6, 20452-20463. [CrossRef] [PubMed]

60. Ishihara, K. Highly lubricated polymer interfaces for advanced artificial hip joints through biomimetic design. Polym. J. 2015, 47, 585-597. [CrossRef]

61. Nie, G.; Li, G.; Wang, L.; Zhang, X. Nanocomposites of polymer brush and inorganic nanoparticles: Preparation, characterization and application. Polym. Chem. 2016, 7, 753-769. [CrossRef]

62. Raj, A.; Wang, M.; Liu, C.; Ali, L.; Karlsson, N.G.; Claesson, P.M.; Dèdinaitè, A. Molecular synergy in biolubrication: The role of cartilage oligomeric matrix protein (COMP) in surface-structuring of lubricin. J. Colloid Interface Sci. 2017, 495, 200-206. [CrossRef] [PubMed] 
63. Suchaoin, W.; Bonengel, S.; Grießinger, J.A.; de Sousa, I.P.; Hussain, S.; Huck, C.W.; Bernkop-Schnürch, A. Novel bioadhesive polymers as intra-articular agents: Chondroitin sulfate-cysteine conjugates. Eur. J. Pharm. Biopharm. 2016, 101, 25-32. [CrossRef] [PubMed]

64. Chang, D.P.; Abu-Lail, N.I.; Guilak, F.; Jay, G.D.; Zauscher, S. Conformational mechanics, adsorption, and normal force interactions of lubricin and hyaluronic acid on model surfaces. Langmuir 2008, 24, 1183-1193. [CrossRef] [PubMed]

65. Chang, D.P.; Abu-Lail, N.I.; Coles, J.M.; Guilak, F.; Jay, G.D.; Zauscher, S. Friction force microscopy of lubricin and hyaluronic acid between hydrophobic and hydrophilic surfaces. Soft Matter 2009, 5, 3438-3445. [CrossRef] [PubMed]

66. Andresen Eguiluz, R.C.; Cook, S.G.; Tan, M.; Brown, C.N.; Pacifici, N.J.; Samak, M.S.; Gourdon, D. Synergistic interactions of a synthetic lubricin-Mimetic with Fibronectin for enhanced Wear Protection. Front. Bioeng. Biotechnol. 2017, 5, 36. [CrossRef] [PubMed]

67. Elsaid, K.A.; Fleming, B.C.; Oksendahl, H.L.; Machan, J.T.; Fadale, P.D.; Hulstyn, M.J.; Jay, G.D. Decreased lubricin concentrations and markers of joint inflammation in the synovial fluid of patients with anterior cruciate ligament injury. Arthritis Rheumatol. 2008, 58, 1707-1715. [CrossRef] [PubMed]

68. Seror, J.; Zhu, L.; Goldberg, R.; Day, A.J.; Klein, J. Supramolecular synergy in the boundary lubrication of synovial joints. Nat. Commun. 2015, 6, 6497. [CrossRef] [PubMed]

69. Hron, J.; Málek, J.; Pustějovská, P.; Rajagopal, K.R. On the modeling of the synovial fluid. Adv. Tribol. 2010, 2010, 104957. [CrossRef]

70. Steele, A.; Bayer, I.S.; Loth, E. Pipe flow drag reduction effects from carbon nanotube additives. Carbon 2014, 77, 1183-1186.

71. Lawrence, A.; Xu, X.; Bible, M.D.; Calve, S.; Neu, C.P.; Panitch, A. Synthesis and characterization of a lubricin mimic (mLub) to reduce friction and adhesion on the articular cartilage surface. Biomaterials 2015, 73, 42-50. [CrossRef] [PubMed]

(C) 2018 by the author. Licensee MDPI, Basel, Switzerland. This article is an open access article distributed under the terms and conditions of the Creative Commons Attribution (CC BY) license (http:/ / creativecommons.org/licenses/by/4.0/). 